\title{
Atmospheric Collapse in Self-Avoiding Walks: A Numerical Study using GARM
}

\author{
J. Alvarez ${ }^{1}$, M. Gara ${ }^{2}$, E. J. Janse van Rensburg ${ }^{1}$ and A. \\ Rechnitzer $^{2}$ \\ ${ }^{1}$ Department of Mathematics and Statistics, York University, Toronto, ON, \\ Canada M3J 1P3 \\ ${ }^{2}$ Department of Mathematics, University of British Columbia, Vancouver, BC, \\ Canada V6T 1 Z2 \\ E-mail: jalvarez@chem.utoronto.ca, rensburg@yorku.ca, andrewr@math.ubc.ca
}

\begin{abstract}
The coil-globule collapse of dilute linear polymers in a poor solvent is generally thought to be a second order phase transition through $\theta$-polymers at the critical point [10]. A common model for the collapse transition of polymers is a lattice self-avoiding walk with a nearest neighbour attraction [10,38]. In this paper we consider an alternative set of models for collapsing linear polymers. In particular, we simulate lattice walks weighted by an atmospheric statistic using the flatGARM-algorithm [40]. These models of walks undergo a collapse transition at a critical value of the parameters of the model. This transition appears to be discontinuous (first order), in contrast to the $\theta$-transition in walks with nearestneighbour contacts. This places our models in a different universality class from the $\theta$-transition in collapsing self-avoiding walks.
\end{abstract}

\section{Introduction}

Linear polymers in a poor solvent collapse into compact balls at the $\theta$-point of the solvent. This continuous phase transition of polymers from extended coils to compact balls is called the $\theta$-transition of linear polymers. It has received significant attention in the polymer physics literature $[10,11,38,45]$ and its scaling theory is generally well understood (see for example references $[10,32,33]$ ).

The $\theta$-transition in linear polymers can be modeled by a self-avoiding walk on a lattice with a nearest neighbour attraction (or "contacts") between non-consecutive vertices of the walk which are adjacent in the lattice. This is the model of collapsing lattice walks $[8,11,32,38,42,45]$. This model has been the subject of numerous numerical studies, but it remains difficult to simulate efficiently, and generally there are reasons to improve on the current estimates of scaling exponents and critical points (see for example references $[2,8,22,42,46]$ ).

The $\theta$-transition in a model of lattice self-avoiding walks with contacts remains unsolved, and relatively little is known rigorously. If $c_{n}(s)$ is the number of selfavoiding walks of length $n$ starting from the origin and with $s$ contacts, then the partition function of this model is

$$
Z_{n}(\beta)=\sum_{s \geq 0} c_{n}(s) e^{\beta s} .
$$




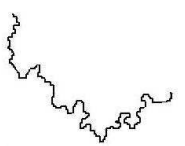

(a)

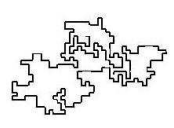

(b)

Figure 1. Collapsing walks: (a) Extended or coiled phase. (b) Compact or globule phase.

The model has a single parameter $\beta$ with critical point $\beta_{k}$; this is the $\theta$-point. The limiting free energy is defined in the usual way: $\mathcal{F}(\beta)=\lim _{n \rightarrow \infty} n^{-1} \log Z_{n}(\beta)$. This limit is known to exist for $\beta \leq 0$, and the typical conformations of the walk in this regime are in an expanded or coiled phase. Figure 1(a) illustrates a walk sampled from this phase.

For positive values of $\beta$, which correspond to a net attractive force between adjacent vertices in the walk, the limiting free energy is not known to exist. The $\theta$-transition corresponds to a singularity in $\mathcal{F}(\beta)$ at the critical point $\beta_{k}$. For more details on this model, see reference [46]. The model goes through the $\theta$-point when $\beta=\beta_{k}$, and for large positive values of $\beta>\beta_{k}$ the attractive forces between vertices collapse the walk. This is the compact or globule phase of the walk [11]. A typical conformation sampled from this phase is depicted in Figure 1(b).

The upper critical dimension for the $\theta$-transition is $d_{c}=3$. In dimensions $d>d_{c}$ the model has mean-field critical scaling and exponents. For example, the crossover exponent of collapse is $\phi=1 / 2$ if $d>d_{c}$. Below $d_{c}$ (in the square lattice) there is considerable variation in the estimates of $\phi$ in the literature. For example, $\phi=0.64 \pm 0.05$ [38] (exact enumeration), $\phi=0.48 \pm 0.07$ [41] (transfer matrix), $\phi=0.52 \pm 0.07[42,43]$ (Monte Carlo), $\phi=0.90 \pm 0.02$ [27] (exact enumeration), $\phi \leq 0.53 \pm 0.004$ [8] (Monte Carlo), and more recently $\phi=0.435 \pm 0.006$ [19] (Monte Carlo), among others. In $d=3$ the critical exponents take on their mean field values, but the scaling in the model is modified by logarithmic terms. Monte Carlo simulations of the model in three dimensions were performed in references [17, 46, 47].

At the $\theta$-point the walk is said to be in $\theta$-conditions. At this point the attractive forces between contacts compensates (to first order) for the self-avoidance of the vertices. The location of the $\theta$-point for square lattice self-avoiding walks has also been estimated numerically as $e^{\beta_{k}}=1.941 \pm 0.047$ [42], $e^{\beta_{k}}=1.95 \pm 0.11$ [27], $e^{\beta_{k}^{*}} \approx 1.90[6], e^{\beta_{k}}=1.93 \pm 0.03[16], e^{\beta_{k}} \approx 1.93[8], e^{\beta_{k}}=1.944 \pm 0.004$ [19], and $e^{\beta_{k}}=1.948 \pm 0.002$ [3]. In the cubic lattice, numerical estimates have also been obtained as $e^{\beta_{k}}=1.317 \pm 0.008$ [47], $e^{\beta_{k}}=1.3204 \pm 0.0055$ [46], and more recently $e^{\beta_{k}}=1.3087 \pm 0.0003$ [17], among others.

The $\theta^{\prime}$-model of polymer collapse was introduced in reference [14]. In this twodimensional model, the collapse transition in linear polymers is modeled by driving a collapse in walks with growing percolation clusters. This model was studied using conformal invariance techniques [48] and exact values are known for its critical exponents - for example the crossover exponent has value $\phi=3 / 7$ [14]. The universality classes of the $\theta$ - and $\theta^{\prime}$-transitions in two dimensions became a subject of considerable interest following the publication of [14]. Initially, there was evidence that 
these models are in the same universality class $[9,14]$, but other studies suggested that this may not be the case; see for example references [8, 15, 27, 28, 34, 35, 44]. (NOTE: changed the rest of this paragraph.) The discrepancies suggesting different universality classes are due to the distinction between ordinary and special surface critical behaviour[14]. Calculations of the $\theta$-model in two dimensions in reference [19] showed that the crossover exponent of this model is consistent with the value $\phi=3 / 7$, providing numerical evidence that the $\theta$-transition is in the same universality class as the $\theta^{\prime}$-transition.

In this paper we (re)consider the collapse of self-avoiding walks from another point of view. Our purpose is to consider the nature of collapse in polymers, and to determine if a collapse transition distinct from the $\theta$-transition can be constructed. We drive the collapse of walks by restricting its degrees of freedom by constraining its atmospheric statistics, which were defined in references [24, 25, 39, 40]. Our results show that in some cases we can induce collapse in walks, and that this transition has the characteristics of a first order transition in the model.

We use the flatGARM-algorithm [40] to simulate collapsing walks where the collapse is driven by fugacities conjugate to the atmospheric statistic. As in the cases of the $\theta$ - and $\theta^{\prime}$ - models, the collapse is essentially driven by confining or limiting the walk to states in conformational space with low degrees of freedom, giving rise to compact globules in the compact phase. Our numerical data suggest that in some cases the transition has the signature of a first order transition: a sharp and apparently discontinuous jump in the energy density.

The atmosphere of a self-avoiding walk can be defined in a number of different ways [24, 39, 40], and we will consider some of them in this paper. The simplest definition can be found in reference [39]; this is the endpoint atmosphere. This atmosphere was used implicitly in even earlier studies involving the Monte Carlo simulation of self-avoiding walks; see for example reference [5]. More general versions of atmospheres have since been defined, in particular the plaquette atmosphere [1, 4, 24] and the generalised atmosphere [40].

In Section 2 we describe endpoint atmospheres and a model of collapsing walks driven by them. We prove that the limiting free energy of that model exists over the entire parameter space and that it is equal to the logarithm of the growth constant of self-avoiding walks.

In Section 3 we describe generalised atmospheres and their distributions. We also describe several models of collapsing walks driven by generalised atmospheres and we prove that the limiting free energy exists for non-negative values of the parameters. The existence of such a limit remains unknown if any of the parameters are negative. This situation is analogous to other collapsing walk models (see references [46, 47]). We carry out a numerical analysis of the phase transition in these models of generalised atmospheres for walks of length 600 . The length of the walks we can analyse is limited by the computational complexity of calculating the generalised atmospheres to obtain the energy of the state, which is $O(n)$, as well as that of calculating the radius of gyration of each walk, which is $O\left(n^{2}\right)$. If the desired final length of the walks is $N$, then the overall complexity from the energy calculations becomes $O\left(N^{2}\right)$, and $O\left(N^{3}\right)$ from the radius of gyration. The data is obtained using the PERM algorithm [17] in its flat histogram sampling implementation (flatPERM [36]) adapted to generalised atmospheres (flatGARM [40]) for the microcanonical ensemble.

In Section 3.3 we focus in particular on a model of collapse driven by a negative generalised atmospheric statistic and argue that there is a strong transition separating 
an expanded phase from a compact phase. Our calculations show that the crossover exponent takes a value consistent with a first order transition. We consider the crossover scaling and present evidence that the scaled internal energy and specific heat curves collapse to universal curves with scaling consistent with first order critical behaviour. We also consider the metric scaling of walks in this model and examine the energy distribution over states to search for coexistence of phases at the critical point. While our data remains very noisy in the low energy (compact) phase, there is some evidence of the coexistence of coil and globule phases in this model.

A model with positive generalised atmospheric collapse is considered in Section 3.4. This model is qualitatively similar to the negative generalised atmospheric collapse model. Then, in Section 3.5 we address a model with neutral generalised atmospheric collapse. This model differs significantly from the other two generalised atmospheric collapse models: there is apparently a continuous crossover between expanded and collapsed regimes but we do not observe scaling or critical behaviour which would signal a critical point. Thus we conclude that there is no phase transition in this model.

We make a few final observations in the conclusions in Section 4.

\section{Endpoint atmospheres}

The endpoint atmosphere of a self-avoiding walk from the origin is defined in terms of the number of ways an edge can be added, removed, or rotated at the end of the walk to obtain another self-avoiding walk. For example, if $\omega$ is a self-avoiding walk of length $n>0$, then a self-avoiding walk $\nu$ of length $n-1 \geq 0$ can be obtained by removing the last edge in $\omega$. We call the last edge in $\omega$ a negative atmospheric edge. The set of such negative atmospheric edges is the negative endpoint atmosphere of $\omega$.

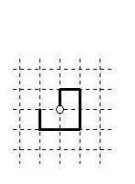

(a)

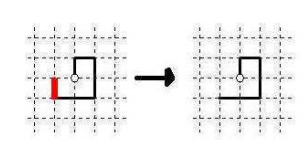

(b)

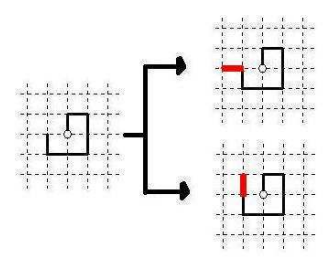

(c)

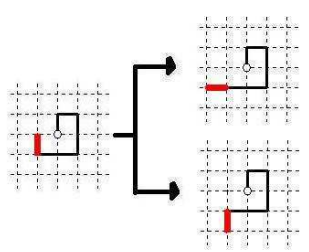

(d)

Figure 2. Examples of endpoint atmospheres for the two-dimensional selfavoiding walk $\omega .(a) \omega .(b) a_{-}^{e}(\omega)=1$. (c) $a_{+}^{e}(\omega)=2$. (d) $a_{0}^{e}(\omega)=2$.

If $\omega$ is a walk from the origin of length $n>0$, then its negative endpoint atmosphere is the set containing only the last edge in $\omega$. If $\omega$ is the trivial walk, then its negative endpoint atmosphere is empty. We denote by $a_{-}^{e}(\omega)$ the size of the negative endpoint atmosphere of $\omega$. Observe that $a_{-}^{e}(\omega)=0$ if $\omega$ is the trivial selfavoiding walk and $a_{-}^{e}(\omega)=1$ otherwise. Figure 2(b) depicts an example of negative endpoint atmospheres.

The positive endpoint atmosphere of a self-avoiding walk $\omega$ is defined by considering the set of edges which can be added at the endpoint of the walk $\omega$ to obtain another self-avoiding walk. The size of the positive endpoint atmosphere is 
denoted by $a_{+}^{e}(\omega)$. In the $d$-dimensional hypercubic lattice $a_{+}^{e}(\omega)=2 d$ for the trivial walk and $0 \leq a_{+}^{e}(\omega) \leq 2 d-1$ otherwise. Figure 2(c) depicts an example of positive endpoint atmospheres. A walk $\omega$ with $a_{+}^{e}(\omega)=0$ is said to be trapped, because it cannot be extended by adding an edge to its endpoint.

A neutral endpoint atmosphere of size $a_{0}^{e}(\omega)$ can be defined by considering the number of ways in which the last edge in the walk $\omega$ can be changed into a new direction to create a different conformation of the same length. For example, if $\omega$ is a walk of length $n=1$, then $a_{0}(\omega)=2 d-1$ in the $d$-dimensional hypercubic lattice, while more generally $0 \leq a_{0}^{e}(\omega) \leq 2 d-2$ for $n>1$, and $a_{0}(\omega)=0$ if $n=0$. Figure $2(d)$ depicts an example of neutral endpoint atmospheres.

A model of walks with endpoint atmospheric collapse can be defined by introducing fugacities $\left\{\beta_{+}, \beta_{0}, \beta_{-}\right\}$conjugate to the atmospheric statistics. The partition function is

$$
Z_{n}\left(\beta_{+}, \beta_{0}, \beta_{-}\right)=\sum_{a_{+}, a_{0}, a_{-}} c_{n}\left(a_{+}^{e}, a_{0}^{e}, a_{-}^{e}\right) e^{\beta_{+} a_{+}^{e}+\beta_{0} a_{0}^{e}+\beta_{-} a_{-}^{e}},
$$

where $c_{n}\left(a_{+}^{e}, a_{0}^{e}, a_{-}^{e}\right)$ is the number of self-avoiding walks of length $n$ with atmospheric statistics $\left(a_{+}^{e}, a_{0}^{e}, a_{-}^{e}\right)$. The limiting free energy of this model is given by $\mathcal{F}\left(\beta_{+}, \beta_{0}, \beta_{-}\right)=\lim _{n \rightarrow \infty} n^{-1} \log Z_{n}\left(\beta_{+}, \beta_{0}, \beta_{-}\right)$and it is a constant function, as we prove in the next theorem.

Theorem 2.1. The limiting free energy $\mathcal{F}\left(\beta_{+}, \beta_{0}, \beta_{-}\right)$for the model of endpoint atmosphere collapsing walks exists for all values of $\left(\beta_{+}, \beta_{0}, \beta_{-}\right)$. Moreover, $\mathcal{F}\left(\beta_{+}, \beta_{0}, \beta_{-}\right) \equiv \log \mu=\kappa$, where $\mu$ is the growth constant and $\kappa$ the connective constant for self-avoiding walks.

Proof. We note that $0 \leq a_{+} \leq 2 d, 0 \leq a_{0} \leq 2 d-1$, and $0 \leq a_{-} \leq 1$. Thus, the partition function is bounded by

$$
\begin{aligned}
& c_{n} e^{3 \min \left\{2 d \beta_{+},(2 d-1) \beta_{0}, \beta_{-}, 0\right\}} \\
& \leq Z_{n}\left(\beta_{+}, \beta_{0}, \beta_{-}\right) \\
& \quad \leq c_{n} e^{3 \max \left\{2 d \beta_{+},(2 d-1) \beta_{0}, \beta_{-}, 0\right\}},
\end{aligned}
$$

where $c_{n}$ is the number of self-avoiding walks of length $n$.

Take logarithms of the above, divide by $n$ and take $n \rightarrow \infty$ to obtain

$$
\begin{aligned}
& \lim _{n \rightarrow \infty}\left(\frac{\log c_{n}}{n}+\frac{3 \min \left\{2 d \beta_{+},(2 d-1) \beta_{0}, \beta_{-}, 0\right\}}{n}\right) \\
& \leq \mathcal{F}\left(\beta_{+}, \beta_{0}, \beta_{-}\right) \\
& \quad \leq \lim _{n \rightarrow \infty}\left(\frac{\log c_{n}}{n}+\frac{3 \max \left\{2 d \beta_{+},(2 d-1) \beta_{0}, \beta_{-}, 0\right\}}{n}\right) .
\end{aligned}
$$

It follows by the squeeze theorem for limits that the free energy exists for all finite values of $\left(\beta_{+}, \beta_{0}, \beta_{-}\right)$, and is equal to $\log \mu$.

\section{Generalised atmospheres}

In this section we consider generalised atmospheres of a self-avoiding walk (see [40]). Suppose that $\omega$ is a self-avoiding walk of length $n$. If $v$ is a vertex in $\omega$, then $\omega$ may be cut into two subwalks $\omega_{1}$ and $\omega_{2}$ at $v$. 
If we consider a lattice edge $e$ incident with $v$, then a new walk of length $n+1$ may be constructed by concatenating the subwalks and $e$ as follows: $\omega^{\prime}=\omega_{1} e \omega_{2}$. Equivalently, one may insert $e$ in $\omega$ at $v$, and then complete the walk by translating $\omega_{2}$ one step in the direction of $e$. If the resulting walk $\omega^{\prime}$ is a self-avoiding walk (of length $n+1$ ), then $e$ is a positive generalised atmospheric edge of $\omega$. In Figure 3 we depict an example of a walk and all its positive generalised atmospheric edges.

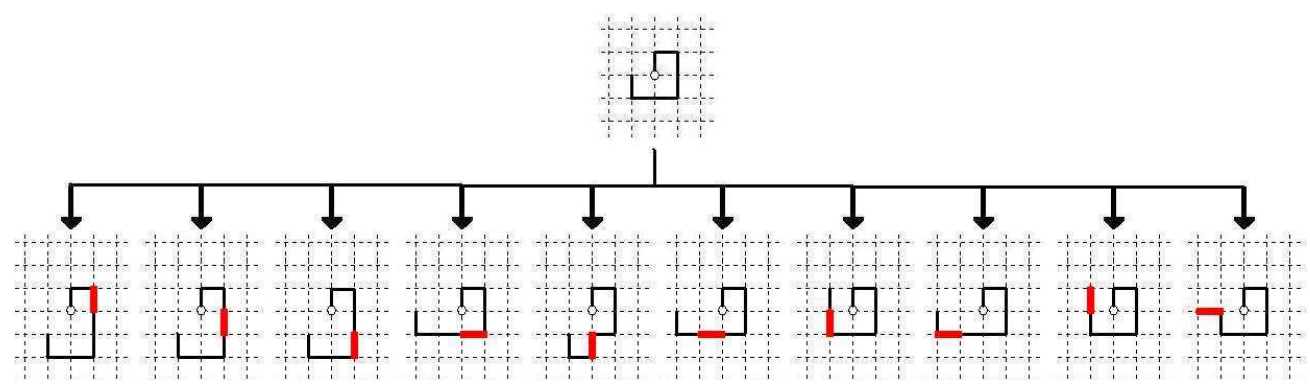

Figure 3. Example of a two-dimensional self-avoiding walk $\omega$ with positive generalised atmosphere $a_{+}^{g}(\omega)=10$. The thicker (red) edge is the edge that is inserted.

The collection of all positive generalised atmospheric edges of $\omega$ is its positive generalised atmosphere and its size is denoted by $a_{+}^{g}(\omega)$. Clearly, $0 \leq a_{+}^{g}(\omega) \leq$ $(2 d-1)(n+1)$ for $n>0$ and if $\omega$ is the trivial walk then $a_{+}^{g}(\omega)=2 d$. Examples of twodimensional self-avoiding walks with small and large positive generalised atmospheres are depicted in Figures 4 and 5.

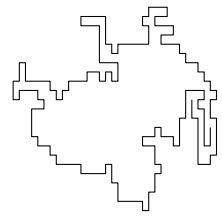

(a)

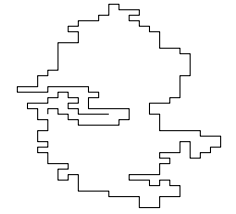

(b)

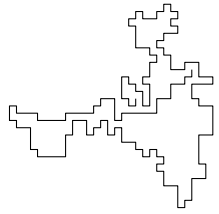

(c)

Figure 4. Two-dimensional self-avoiding walks of length $n=200$ with small positive generalised atmospheres per vertex. $(a) a_{+}^{g}(\omega)=8,(b) a_{+}^{g}(\omega)=7,(c)$ $a_{+}^{g}(\omega)=15$.

If $\omega$ is a walk of length $n$, and $e$ is an edge in $\omega$, then $e$ is a negative generalised atmospheric edge in $\omega$ if the walk $\omega^{\prime \prime}$, obtained by contracting $e$ in $\omega$, is self-avoiding. Figure 6 depicts an example of a walk and all its negative generalised atmospheric edges.

The collection of all negative generalised atmospheric edges of $\omega$ is the negative generalised atmosphere, and its size is denoted by $a_{-}^{g}(\omega)$. Clearly, $2 \leq a_{-}^{g}(\omega) \leq n$, if $\omega$ is a walk of length at least 2 edges. If $\omega$ is a walk of length 1 , then $a_{-}^{g}(\omega)=1$ and if $\omega$ is the trivial walk, then $a_{-}^{g}(\omega)=0$. Examples of two-dimensional walks with small and large negative generalised atmospheres are depicted in Figures 7 and 8.

Suppose that $e_{i}$ and $e_{i+1}$ are consecutive edges in a walk $\omega$. That is, $\omega=$ 


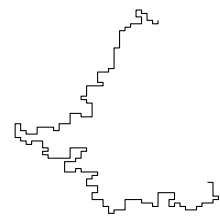

(a)

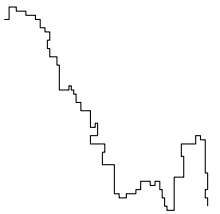

(b)

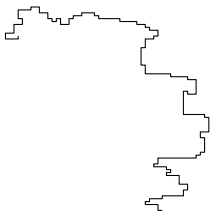

(c)

Figure 5. Two-dimensional self-avoiding walks of length $n=200$ with large positive generalised atmospheres per vertex. $(a) a_{+}^{g}(\omega)=448,(b) a_{+}^{g}(\omega)=519$, (c) $a_{+}^{g}(\omega)=503$.

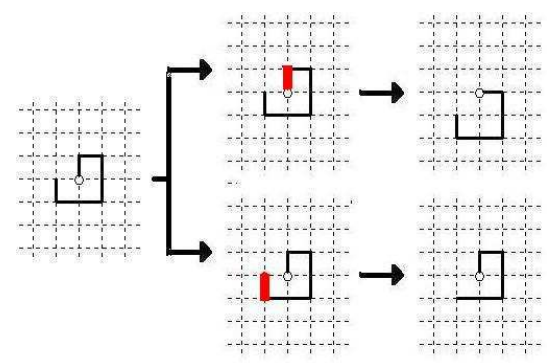

Figure 6. Example of a two-dimensional self-avoiding walk $\omega$ with negative generalised atmosphere $a_{-}^{g}(\omega)=2$. The thicker (red) edge is the edge that is removed.

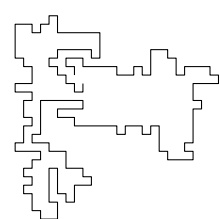

(a)

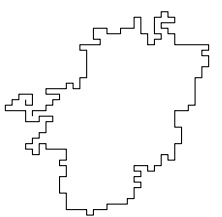

(b)

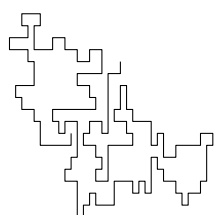

(c)

Figure 7. Two-dimensional self-avoiding walks of length $n=200$ with small negative generalised atmospheres per vertex. $(a) a_{-}^{g}(\omega)=6,(b) a_{-}^{g}(\omega)=7,(c)$ $a_{-}^{g}(\omega)=9$.

$\omega_{1} e_{i} e_{i+1} \omega_{2}$, where $\omega_{1}$ and $\omega_{2}$ are subwalks, possibly trivial. If the walk $\omega^{\prime \prime \prime}=$ $\omega_{1} e_{i+1} e_{i} \omega_{2}$ is self-avoiding and $\omega^{\prime \prime \prime} \neq \omega$ (this occurs when $e_{i} \perp e_{i+1}$ ), then the pair $\left(e_{i}, e_{i+1}\right)$ is a pair of neutral atmospheric edges. Figure 9 depicts an example of a walk and all its neutral generalised atmospheric edges.

The neutral atmosphere of $\omega$ is the collection of all pairs of neutral atmospheric edges, and it has size $a_{0}^{g}(\omega)$. Clearly, $0 \leq a_{0}^{g}(\omega) \leq n-1$ for $n>0$ and if $\omega$ is the trivial walk of length $n=0$ then $a_{0}^{g}(\omega)=0$. Examples of two-dimensional walks with large and small neutral atmospheres are depicted in Figures 10 and 11. 


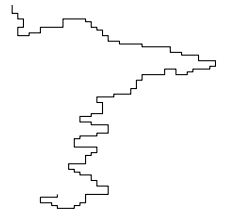

(a)

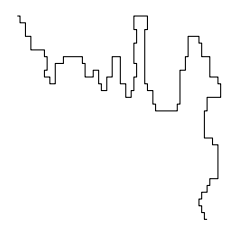

(b)

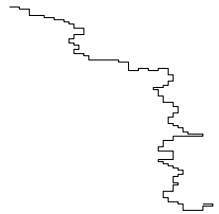

(c)

Figure 8. Two-dimensional self-avoiding walks of length $n=200$ with large negative generalised atmospheres per vertex. $(a) a_{-}^{g}(\omega)=200,(b) a_{-}^{g}(\omega)=198$, (c) $a_{-}^{g}(\omega)=195$.

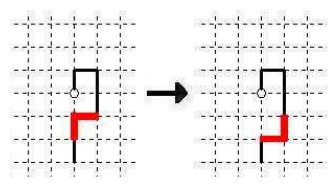

Figure 9. Example of a two-dimensional self-avoiding walk $\omega$ with neutral generalised atmosphere $a_{0}^{g}(\omega)=1$. The thicker (red) edges constitute the pair of edges that is reordered.

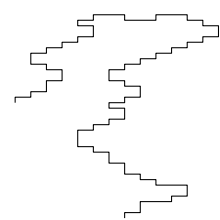

(a)

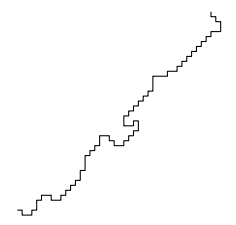

(b)

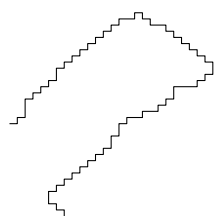

(c)

Figure 10. Two-dimensional self-avoiding walks of length $n=100$ with large neutral generalised atmospheres per vertex. $(a) a_{0}^{g}(\omega)=76,(b) a_{0}^{g}(\omega)=76,(c)$ $a_{0}^{g}(\omega)=83$.

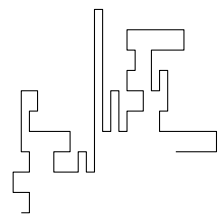

(a)

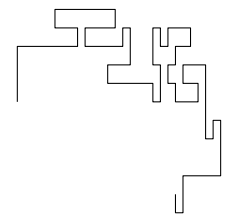

(b)

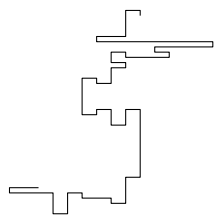

(c)

Figure 11. Two-dimensional self-avoiding walks of length $n=100$ with small neutral generalised atmospheres per vertex. $(a) a_{0}^{g}(\omega)=1,(b) a_{0}^{g}(\omega)=5,(c)$ $a_{0}^{g}(\omega)=8$. 


\subsection{Atmospheric distributions in walks}

The mean size of an atmospheric statistic over walks of length $n$ is denoted by $\left\langle a_{\bullet}^{g}\right\rangle_{n}$, where $\bullet=+,-, 0$. The bounds on the sizes of generalised atmospheres suggest that $\left\langle a_{\bullet}^{g}\right\rangle_{n} \sim C n$ for some constant $C$ depending on the lattice and on the type of atmosphere. In the case of endpoint atmospheres, $C=0$, but it appears that $C>0$ for generalised atmospheres. However we have been unable to prove this. An argument in references $[24,39]$ shows that

$$
\frac{\left\langle a_{+}^{g}\right\rangle_{n}}{\left\langle a_{-}^{g}\right\rangle_{n}}=\frac{c_{n+1}}{c_{n}}
$$

Since $c_{n+1} / c_{n}$ is thought to approach the growth constant $\mu$ of self-avoiding walks as $n \rightarrow \infty$, it follows that measuring the constant $C$ for positive and negative atmospheres allows us to estimate $\mu$.

Define $c_{n}\left(a_{\bullet}^{g}\right)$ to be the number of self-avoiding walks of length $n$ from the origin with atmospheric statistic of size $a_{\bullet}^{g}$. For example, $c_{n}\left(a_{+}^{g}\right)$ is the number of walks of length $n$ and with positive atmosphere of size $a_{+}^{g}$. Then, the mean value of the positive generalised atmosphere is defined by $\left\langle a_{+}^{g}\right\rangle_{n}=\left[\sum a_{+}^{g} c_{n}\left(a_{+}^{g}\right)\right] / c_{n}$, and similarly for $a_{-}^{g}$ and $a_{0}^{g}$.

We estimated the mean sizes of generalised atmospheres in the square lattice by generating walks using the flatGARM-algorithm [40]. Analysing our data gives the following results:

$$
\begin{aligned}
& \left\langle a_{-}^{g}\right\rangle_{n} \approx 0.82(n+0.4+o(1)) \\
& \left\langle a_{+}^{g}\right\rangle_{n} \approx 2.17(n+1.7+o(1)) \\
& \left\langle a_{0}^{g}\right\rangle_{n} \approx 0.38(n-0.9+o(1))
\end{aligned}
$$

Observe that $[2.17 / 0.82]=2.646 \approx \mu$, as one would expect.

The peak of the distribution of $c_{n}\left(a_{\bullet}^{g}\right)$ as a function of $a_{\bullet}^{g}$ should increase proportionally to $c_{n}$ as $n$ increases, so $c_{n}\left(a_{\bullet}^{g}\right) / c_{n}$ would be a normalised distribution of atmospheres. Since $0 \leq a_{\bullet}^{g} \leq K n$ for some constant $K$, the support of the distribution also increases at most linearly in $n$, and thus one would expect that for some $\epsilon \in[0,1]$, plots of $\left[c_{n}\left(a_{\bullet}^{g}\right) / c_{n}\right] n^{\epsilon}$ against $\left(a_{\bullet}^{g} / n-C\right) n^{1-\epsilon}$ should collapse to a universal curve which describes the scaling of $c_{n}\left(a_{\bullet}^{g}\right)$. The value of $C$ is given in equations 4,5 and 6. For example, one would expect that for some value of $\epsilon,\left[c_{n}\left(a_{-}^{g}\right) / c_{n}\right] n^{\epsilon}$ plotted against $\left(a_{-}^{g} / n-C\right) n^{1-\epsilon}$ would give a curve independent of $n$ if $C \approx 0.82$. Our best results are obtained for $C=0.81$ and for $\epsilon=2 / 3$ in Figure 12, for values of

$n=32,45,64,91,128,181$ and 256 (these rounded values of powers of $\sqrt{2}$ provide a good range of $n$ values). The family of curves collapse to a single universal curve describing the scaling of the distribution of negative generalised atmospheres in square lattice walks.

Similar data have been obtained for positive generalised atmospheres and are plotted in Figure 13(a). In this case we obtain our best results when $C=2.08$, which is slightly smaller than the $C=2.17$ obtained above. The value of $\epsilon$ is $\epsilon=2 / 3$. Thus, the peak height of the distribution $\left[c_{n}\left(a_{+}^{g}\right) / c_{n}\right]$ grows as $n^{2 / 3}$ while its width decreases as $n^{1 / 3}$, for both positive and negative generalised atmospheres. Additionally note that the shapes of the limiting distributions of positive and negative atmospheres are very similar.

In contrast, when we fitted the distribution of neutral atmospheres we found the best data collapse when $\epsilon=1 / 2$ and $C=0.38$ (see Figure 13(b)). Furthermore, the 


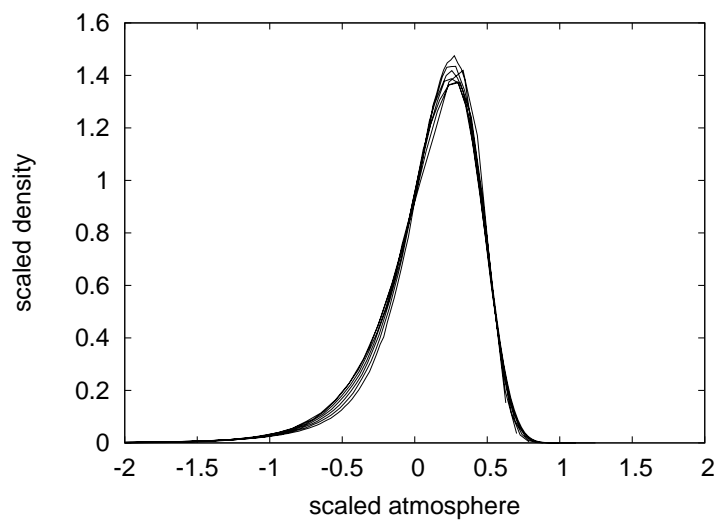

Figure 12. The scaled distribution of negative generalised atmospheres for twodimensional walks. We have plotted $\left[\frac{c_{n}\left(a_{-}^{g}\right)}{c_{n}}\right] n^{2 / 3}$ against $\left(a_{-}^{g} / n-0.81\right) n^{1 / 3}$ for $n=32,45,64,91,128,181,256$.

collapsed curve is quite symmetric about its peak, unlike the asymmetric distributions observed for the positive and negative atmospheres.

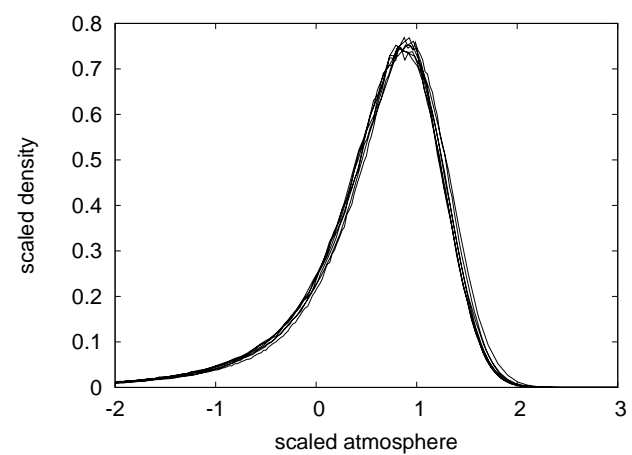

(a)

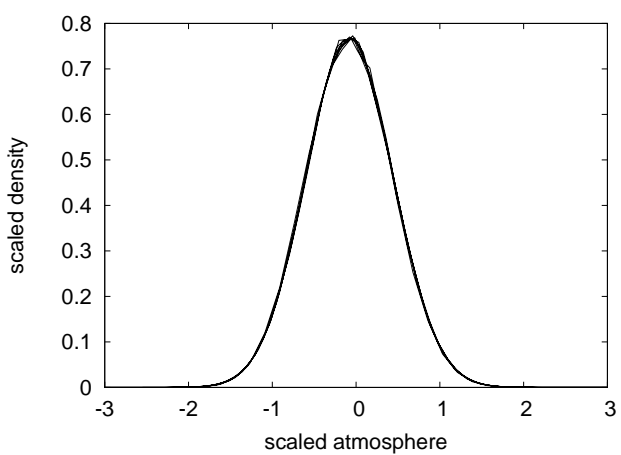

(b)

Figure 13. The scaled distribution $\left[\frac{c_{n}\left(a_{\bullet}^{g}\right)}{c_{n}}\right] n^{\epsilon}$ plotted against $\left(a_{\bullet}^{g} / n-C\right) n^{1-\epsilon}$ for: (a) positive generalised atmospheres, where $\epsilon=2 / 3$ and $C=2.08$. (b) neutral generalised atmospheres, where $\epsilon=1 / 2$ and $C=0.38$. The curves correspond to walks of length $n=32,45,64,91,128,181,256$.

\subsection{Atmospheric collapse}

In this section we consider models of walks with a collapse transition driven by generalised atmospheric statistics. Let $c_{n}\left(a_{+}^{g}, a_{0}^{g}, a_{-}^{g}\right)$ be the number of self-avoiding walks from the origin with generalised atmospheric statistics $\left(a_{+}^{g}, a_{0}^{g}, a_{-}^{g}\right)$. The 
partition function of this model is

$$
Z_{n}\left(\beta_{+}, \beta_{0}, \beta_{-}\right)=\sum_{a_{+}^{g}, a_{0}^{g}, a_{-}^{g}} c_{n}\left(a_{+}^{g}, a_{0}^{g}, a_{-}^{g}\right) e^{\beta_{+} a_{+}^{g}+\beta_{0} a_{0}^{g}+\beta_{-} a_{-}^{g}} .
$$

We prove the existence of the limiting free energy in this model, $\mathcal{F}\left(\beta_{+}, \beta_{0}, \beta_{-}\right)=$ $\lim _{n \rightarrow \infty} n^{-1} \log Z_{n}\left(\beta_{+}, \beta_{0}, \beta_{-}\right)$, by using unfolded walks (see reference [20]).

Let $\omega$ be a self-avoiding walk with vertices $\left\{v_{i}\right\}_{i=0}^{n}$. Suppose that the coordinates of a vertex $v$ are given by $(X(v), Y(v), \ldots, Z(v))$ in $d$ dimensions, with $X(v)$ the first coordinate, $Y(v)$ the second coordinate, and $Z(v)$ the last (or d-th) coordinate of $v$. In two dimensions, $Y(v) \equiv Z(v)$. The self-avoiding walk is $X$-unfolded (or just unfolded) if $X\left(v_{0}\right) \leq X\left(v_{i}\right)<X\left(v_{n}\right)$ for all $i=1,2, \ldots, n-1$. We say that a walk is strongly unfolded if the last inequalities are strict: $X\left(v_{0}\right)<X\left(v_{i}\right)<X\left(v_{n}\right)$ for all $i=1,2, \ldots, n-1$.

Two strongly unfolded walks $\omega_{1}$ and $\omega_{2}$ can be concatenated by translating $\omega_{2}$ so that its first vertex coincides with the last vertex in $\omega_{1}$. The resulting walk $\omega=\omega_{1} \omega_{2}$ is itself strongly unfolded, and the atmospheric statistics of the walks $\omega_{1}$ and $\omega_{2}$ are almost additive under this construction (the positive generalised atmosphere decreases by exactly $2 d-1$, while the negative and neutral atmospheres are additive).

Define $c_{n}^{\ddagger}\left(a_{+}^{g}, a_{0}^{g}, a_{-}^{g}\right)$ to be the number of strongly unfolded self-avoiding walks of length $n$ from the origin with generalised atmospheric statistics $\left(a_{+}^{g}, a_{0}^{g}, a_{-}^{g}\right)$. The partition function of these walks is

$$
Z_{n}^{\ddagger}\left(\beta_{+}, \beta_{0}, \beta_{-}\right)=\sum_{a_{+}^{g}, a_{0}^{g}, a_{-}^{g}} c_{n}^{\ddagger}\left(a_{+}^{g}, a_{0}^{g}, a_{-}^{g}\right) e^{\beta_{+} a_{+}^{g}+\beta_{0} a_{0}^{g}+\beta_{-} a_{-}^{g}} .
$$

The existence of a limiting free energy for the model of strongly unfolded walks is established by the following theorem.

Lemma 3.1. The limiting free energy of strongly unfolded self-avoiding walks

$$
\mathcal{F}^{\ddagger}\left(\beta_{+}, \beta_{0}, \beta_{-}\right)=\lim _{n \rightarrow \infty} \frac{1}{n} \log Z_{n}^{\ddagger}\left(\beta_{+}, \beta_{0}, \beta_{-}\right) .
$$

exists for all $\beta_{+}, \beta_{0}, \beta_{-}<\infty$.

Proof. We first determine an upper bound on the partition function. As indicated in Section 3, the number of positive atmospheres is bounded above by $(2 d-1)(n+1)$, the number of neutral atmospheres by $n-1$, and the number of negative atmospheres by $n$.

Two strongly unfolded self-avoiding walks can be concatenated by identifying the last vertex of the first with the first vertex of the second. This gives again a strongly unfolded walk. We note that the negative and neutral generalised atmospheres are additive under this construction, but the positive generalised atmosphere of the resulting walk decreased by $(2 d-1)$ over the sum of the positive generalised atmospheres of the two given strongly unfolded walks. That is, since this construction is an injection, one obtains

$$
\sum_{\hat{a}_{+}, \hat{a}_{0}, \hat{a}_{-}} c_{m}^{\ddagger}\left(\hat{a}_{+}, \hat{a}_{0}, \hat{a}_{-}\right) c_{n}^{\ddagger}\left(a_{+}^{g}-\hat{a}_{+}, a_{0}^{g}-\hat{a}_{0}, a_{-}^{g}-\hat{a}_{-}\right) \leq c_{n+m}^{\ddagger}\left(a_{+}^{g}-(2 d-1), a_{0}^{g}, a_{-}^{g}\right) .
$$

Multiply both sides of the last inequality by $e^{\left(a_{+}^{g}-(2 d-1)\right) \beta_{+}+\beta_{0} a_{0}^{g}+\beta_{-} a_{-}^{g}}$ and sum over $a_{+}^{g}, a_{0}^{g}, a_{-}^{g}$, to get the inequality

$$
\left[\hat{Z}_{n}^{\ddagger}\left(\beta_{+}, \beta_{0}, \beta_{-}\right)\right]\left[\hat{Z}_{m}^{\ddagger}\left(\beta_{+}, \beta_{0}, \beta_{-}\right)\right] \leq\left[e^{-(2 d-1) \beta_{+}} \hat{Z}_{n+m}^{\ddagger}\left(\beta_{+}, \beta_{0}, \beta_{-}\right)\right] .
$$


That means that $\left[e^{(2 d-1) \beta_{+}} \hat{Z}_{n}^{\ddagger}\left(\beta_{+}, \beta_{0}, \beta_{-}\right)\right]$is a supermultiplicative function of $n$.

The number of strongly unfolded self-avoiding walks of length $n$ is bounded above by $(2 d-1)^{n-2}$ in the square lattice because each step, except the first and the last, can be in any of $2 d-1$ lattice directions. With the bounds on the sizes of the atmospheres above, this gives the upper bound

$$
Z_{n}^{\ddagger}\left(\beta_{+}, \beta_{0}, \beta_{-}\right) \leq(2 d-1)^{n-2} e^{\max \left\{0,(2 d-1)(n+1) \beta_{+}\right\}+\max \left\{0,(n-1) \beta_{0}\right\}+\max \left\{0, n \beta_{-}\right\}},
$$

for any $\beta_{+}, \beta_{0}, \beta_{-}<\infty$ on the partition function. Together with the supermultiplicative inequality for $\left[e^{(2 d-1) \beta_{+}} \hat{Z}_{\dot{n}}^{\ddagger}\left(\beta_{+}, \beta_{0}, \beta_{-}\right)\right]$this proves that the limiting free energy $\hat{\mathcal{F}}^{\ddagger}\left(\beta_{+}, \beta_{0}, \beta_{-}\right)$exists for all $\beta_{+}, \beta_{0}, \beta_{-}<\infty$, see for example reference [21].

The existence of a limiting free energy of atmospherically driven collapsing walks can be demonstrated for non-negative values of the parameters $\beta_{\bullet}$. For negative values of $\beta_{\bullet}$, we will assume that the model has a limiting free energy; this situation is analogous to (nearest neighbour contact driven) collapsing walk models (see references $[46,47]$ ), where the existence of a limiting free energy is generally believed to exist for attractive forces but remains unproved.

Theorem 3.2. The limiting free energy

$$
\mathcal{F}\left(\beta_{+}, \beta_{0}, \beta_{-}\right)=\lim _{n \rightarrow \infty} \frac{1}{n} \log Z_{n}\left(\beta_{+}, \beta_{0}, \beta_{-}\right) .
$$

exists for all $\beta_{+}, \beta_{0}, \beta_{-} \geq 0$. Furthermore, $\mathcal{F}\left(\beta_{+}, \beta_{0}, \beta_{-}\right)=\mathcal{F}^{\ddagger}\left(\beta_{+}, \beta_{0}, \beta_{-}\right)$for all $\beta_{+}, \beta_{0}, \beta_{-} \geq 0$.

Proof. The proof proceeds similarly to the Hammersley-Welsh argument relating walks to unfolded walks - details of the construction can be found in reference [20].

Observe that $c_{n}^{\ddagger}\left(a_{+}^{g}, a_{0}^{g}, a_{-}^{g}\right) \leq c_{n}\left(a_{+}^{g}, a_{0}^{g}, a_{-}^{g}\right)$. Therefore, the partition functions are similarly related: $Z_{n}^{\ddagger}\left(\beta_{+}, \beta_{0}, \beta_{-}\right) \leq Z_{n}\left(\beta_{+}, \beta_{0}, \beta_{-}\right)$.

Next, a walk can be strongly unfolded by first unfolding the walk as in reference $[20]$ and then by adding two new edges in the $X$-direction; one edge on each endpoint of the walk.

The strongly unfolding of a walk increases the generalised atmospheric statistics in general. To see this consider the two subwalks involved in a single unfolding operation, then any atmosphere that was present in the full walk is still present in the subwalks, and reflecting a subwalk to unfold the full walk will not change this fact. Therefore, if the strongly unfolded walk $\omega^{\ddagger}$ (of length $n+2$ ) is obtained by unfolding the walk $\omega$, then $a_{+}^{g}(\omega) \leq a_{+}^{g}\left(\omega^{\ddagger}\right), a_{0}^{g}(\omega) \leq a_{0}^{g}\left(\omega^{\ddagger}\right)$, and $a_{-}^{g}(\omega) \leq a_{-}^{g}\left(\omega^{\ddagger}\right)$. This can be seen by noting that if $e$ is a positive or negative atmospheric edge in $\omega$, then it is also a positive or negative atmospheric edge in $\omega^{\ddagger}$. A similar observation is valid for neutral atmospheric edges.

Therefore, it follows that $e^{\beta_{+} a_{+}^{g}(\omega)+\beta_{0} a_{0}^{g}(\omega)+\beta_{-} a_{-}^{g}(\omega)} \leq e^{\beta_{+} a_{+}^{g}\left(\omega^{\ddagger}\right)+\beta_{0} a_{0}^{g}\left(\omega^{\ddagger}\right)+\beta_{-} a_{-}^{g}\left(\omega^{\ddagger}\right)}$ for all $\beta_{+}, \beta_{0}, \beta_{-} \geq 0$. Summing over all walks and strongly unfolded walks, while noting that at most $e^{O(\sqrt{n})}$ walks are unfolded to the same strongly unfolded walk, it follows that

$$
\begin{aligned}
Z_{n}\left(\beta_{+}, \beta_{0}, \beta_{-}\right) & =\sum_{\omega} e^{\beta_{+} a_{+}^{g}(\omega)+\beta_{0} a_{0}^{g}(\omega)+\beta_{-} a_{-}^{g}(\omega)} \\
& \leq \sum_{\omega} e^{\beta_{+} a_{+}^{g}\left(\omega^{\ddagger}\right)+\beta_{0} a_{0}^{g}\left(\omega^{\ddagger}\right)+\beta_{-} a_{-}^{g}\left(\omega^{\ddagger}\right)}
\end{aligned}
$$




$$
\begin{aligned}
& \leq e^{O(\sqrt{n})} \sum_{\omega^{\ddagger}} e^{\beta_{+} a_{+}^{g}\left(\omega^{\ddagger}\right)+\beta_{0} a_{0}^{g}\left(\omega^{\ddagger}\right)+\beta_{-} a_{-}^{g}\left(\omega^{\ddagger}\right)} \\
& =e^{O(\sqrt{n})} Z_{n+2}^{\ddagger}\left(\beta_{+}, \beta_{0}, \beta_{-}\right),
\end{aligned}
$$

because the resulting strongly unfolded walks have length $n+2$.

In other words, it follows that

$$
Z_{n}^{\ddagger}\left(\beta_{+}, \beta_{0}, \beta_{-}\right) \leq Z_{n}\left(\beta_{+}, \beta_{0}, \beta_{-}\right) \leq e^{O(\sqrt{n})} Z_{n+2}^{\ddagger}\left(\beta_{+}, \beta_{0}, \beta_{-}\right) .
$$

Take logarithms on both sides, divide by $n$ on both sides, and let $n \rightarrow \infty$ to get $\mathcal{F}\left(\beta_{+}, \beta_{0}, \beta_{-}\right)=\mathcal{F}^{\ddagger}\left(\beta_{+}, \beta_{0}, \beta_{-}\right)$.

In the remainder of this section, we analyse three special cases of this generalised atmospheric collapse model: one related only to negative atmospheres, another related only to positive atmospheres, and one related only to neutral atmospheres.

\subsection{Negative atmospheric collapse in walks}

The flatGARM algorithm [40] was implemented to simulate self-avoiding walks while tracking negative atmospheric statistics. flatGARM is a kinetic sampling algorithm with pruning and enrichment moves similar to flatPERM $[17,36]$. We grew walks of lengths up to $n=600$ and collected data in increments of 10 . A total of $3.74 \times 10^{5}$ walks were started, and the pruning and enrichment processes eventually produced a sample of $2.23 \times 10^{10}$ walks for data analysis, of which $2.12 \times 10^{8}$ walks reached the full length. The number of walks that reach the full length is larger than the number of started walks because the number of walks at each length $n$ is dependent of the walk length in the flatGARM algorithm. These walks were analysed to determine free energies and metric properties. The length of the walks analysed is limited by the computational complexity of calculating the generalised atmospheres to obtain the energy of the state at each walk length. If the desired final length of the walks is $N$, then the overall complexity from the energy calculations becomes $O\left(N^{2}\right)$, and $O\left(N^{3}\right)$ from the radius of gyration.

The partition function of walks with negative atmospheric collapse is given by $Z_{n}\left(\beta_{-}\right) \equiv Z_{n}\left(0,0, \beta_{-}\right)$as in equation 7 . We define $c_{n}\left(a_{-}^{g}\right)$ to be the number of walks of length $n$ with negative generalised atmosphere equal to $a_{-}^{g}$. Then $Z_{n}\left(\beta_{-}\right)=$

$\sum_{a_{-}^{g}} c_{n}\left(a_{-}^{g}\right) e^{\beta_{-} a_{-}^{g}}$. For large negative values of $\beta_{-}, Z_{n}\left(\beta_{-}\right)$will be dominated by walks with small $a_{-}^{g}$, and for large positive values of $\beta_{-}$, it will be dominated by walks with large $a_{-}^{g}$. Since walks with large negative atmospheres tend to assume conformations which appear expanded and walks with small negative atmospheres tend to assume conformations which appear to be more compact, one expects two regimes in this model: A regime of expanded walks for large positive $\beta_{-}$and a regime of compact walks for large negative $\beta_{-}$. For convenience we will refer to these as the coil and globule phases - indeed we find a phase transition between them and additionally see that the metric exponents indicate a transition from an extended phase to a more compact phase. Typical conformations of two-dimensional walks with small and large negative atmospheres are depicted in Figures 7 and 8 .

The intensive free energy of this model is defined by

$$
\mathcal{F}_{n}\left(\beta_{-}\right)=\frac{1}{n} \log Z_{n}\left(\beta_{-}\right),
$$


and the limiting free energy $\mathcal{F}\left(\beta_{-}\right)=\lim _{n \rightarrow \infty} \mathcal{F}_{n}\left(\beta_{-}\right)$exists for all values of $\beta_{-} \geq 0$; this follows from Theorem 3.2 because $\mathcal{F}_{n}\left(\beta_{-}\right) \equiv \mathcal{F}_{n}\left(0,0, \beta_{-}\right)$. Existence of a limiting free energy in this model for $\beta_{-}<0$ remains an open question: see reference [46] for a similar situation for collapsing walks in the $\theta$-model.

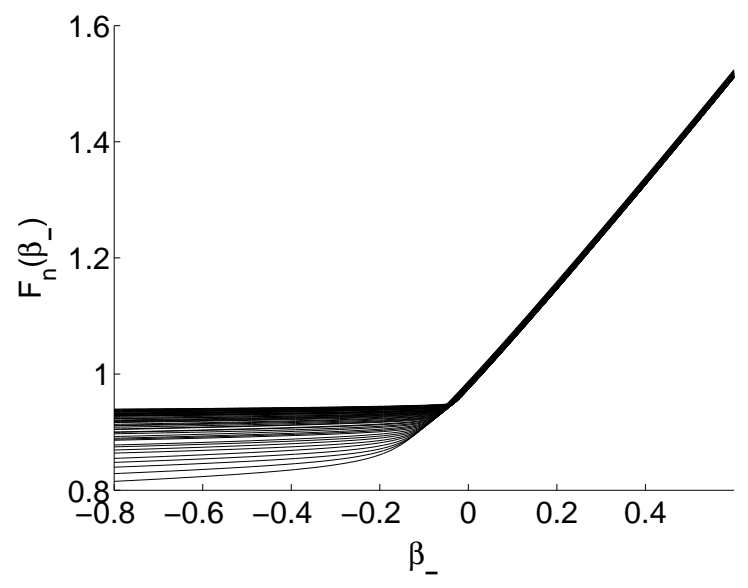

Figure 14. Intensive free energy, $\mathcal{F}_{n}\left(\beta_{-}\right)$of collapsing walks driven by a negative atmospheric fugacity $\beta_{-}$. For negative values of $\beta_{-}$the curves increase with $n$, while for large positive values of $\beta_{-}$, the curves appears to have stabilised along a line. The sharp transition at $\beta_{-}^{*} \approx-0.01$ corresponds to a coil-globule collapse transition in this model.

In Figure 14 we plot the intensive free energy $\mathcal{F}_{n}\left(\beta_{-}\right)$for two-dimensional walks. There appears to be a sharp transition at a critical point of the fugacity $\beta_{-}=\beta_{-}^{*} \approx-0.01$. The sharp turn in $\mathcal{F}_{n}\left(\beta_{-}\right)$at $\beta_{-}^{*}$ from an almost horizontal line to an increasing limiting curve suggests a discontinuous (first order) collapse transition.

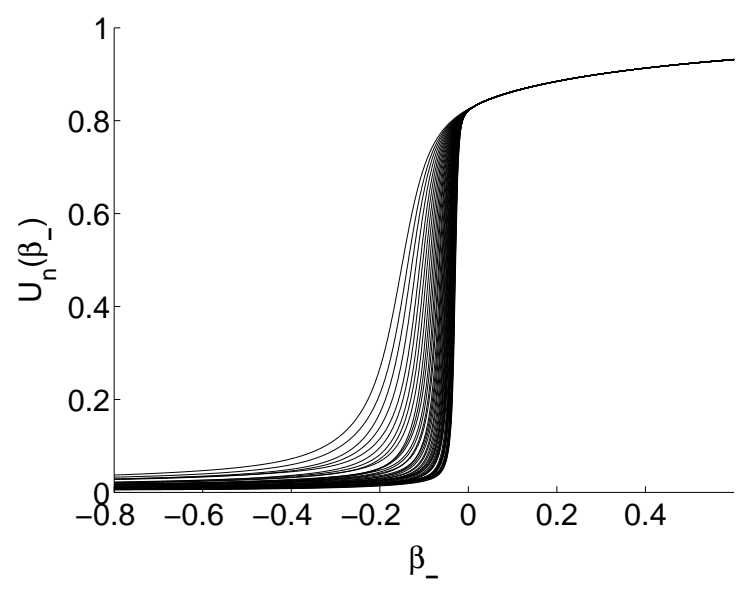

Figure 15. Internal energy per monomer, $U_{n}\left(\beta_{-}\right)$for $n \in[100,600]$ in intervals of 10. The curves approach a limiting curve with a jump discontinuity with increasing $n$, consistent with a first order coil-globule collapse in this model.

The nature of the transition may be examined by considering the internal energy 
of the model. This is given by $\left\langle a_{-}^{g}\right\rangle_{n}=\frac{d}{d \beta_{-}} \mathcal{F}_{n}\left(\beta_{-}\right)$, while the energy density (internal energy per monomer) is defined by $U_{n}\left(\beta_{-}\right)=\left\langle a_{-}^{g}\right\rangle_{n} / n$. We plot the energy density in Figure 15. Observe that the energy density accumulates with increasing $n$ on a limiting curve with a jump discontinuity consistent with a first order phase transition at $\beta_{-}^{*}$.

The transition should also be visible in the specific heat of the model, defined by the variance of the negative atmospheric statistic per monomer:

$$
C_{n}\left(\beta_{-}\right)=\left(\left\langle\left(a_{-}^{g}\right)^{2}\right\rangle_{n}-\left\langle a_{-}^{g}\right\rangle_{n}^{2}\right) / n=\frac{1}{n} \frac{\partial^{2} \mathcal{F}_{n}\left(\beta_{-}\right)}{\partial \beta_{-}^{2}}
$$

The specific heat for finite length $n$ should diverge near the critical point with increasing $n$. In Figure $16 C_{n}\left(\beta_{-}\right)$is plotted as a function of $\beta_{-}$for values of $n \in[100,600]$ separated by intervals of 10 .

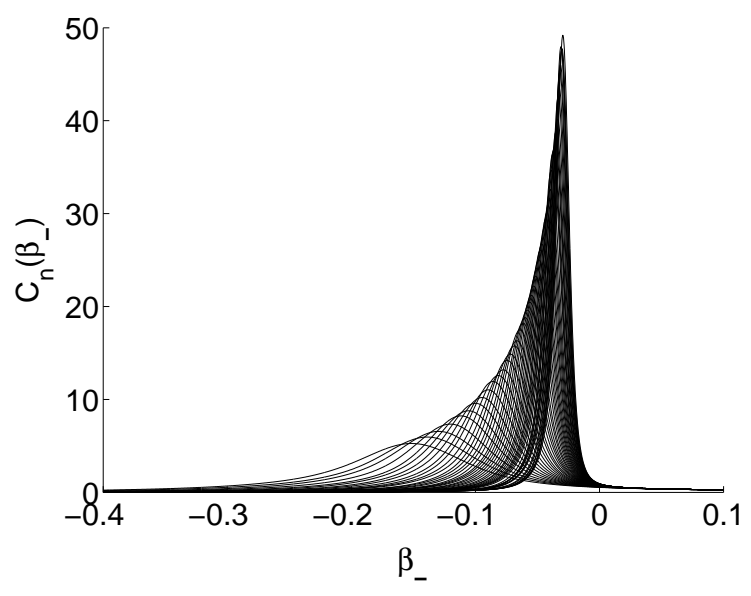

Figure 16. Specific heat, $C_{n}\left(\beta_{-}\right)$. The height of the peak in the specific heat increases with $n$, and moves to the right.

Critical scaling of the specific heat in models of collapsing polymers (see reference [11]) is described by tricritical exponents $\alpha$ (the specific heat exponent), $\phi$ (the crossover exponent) and a universal scaling function $f$ such that:

$$
C_{n}\left(\beta_{-}\right) \sim n^{2 \phi-1} f\left(n^{\phi}\left(\beta_{-}-\beta_{-}^{*}\right)\right)
$$

where $2-\alpha=1 / \phi$ is a hyperscaling relation relating the singular behaviour of $C_{n}$ with crossover scaling in the limiting free energy as $\beta_{-} \nearrow \beta_{-}^{*}$. The height of the peak in the specific heat grows as $A n^{2 \phi-1}$, for some real positive constant $A=f(0)$ and a real crossover exponent $\phi$. For continuous transitions, $0<\phi<1$, while $\phi=1$ would be consistent with a first order transition in the model.

We examined the scaling of $C_{n}\left(\beta_{-}\right)$to determine $\phi$ from the data in Figure 16 for $n \geq 200$. The resulting fit of the peak height against $n$ suggest that $A \approx 0.0176$ and $\phi \approx 1.12$, and it is plotted in Figure 17(a) along with the actual values of the peak heights. These results are subject to strong corrections to scaling in the data, and normally one would not expect values of $\phi$ larger than one.

The appearance of our data suggest a first order transition, and we proceeded by assuming that $\phi=1$ and that the height of the peak in the specific heat grows as $A n^{2 \phi-1}+\hat{a}$ to account for the strong small $n$ corrections. This produced the 


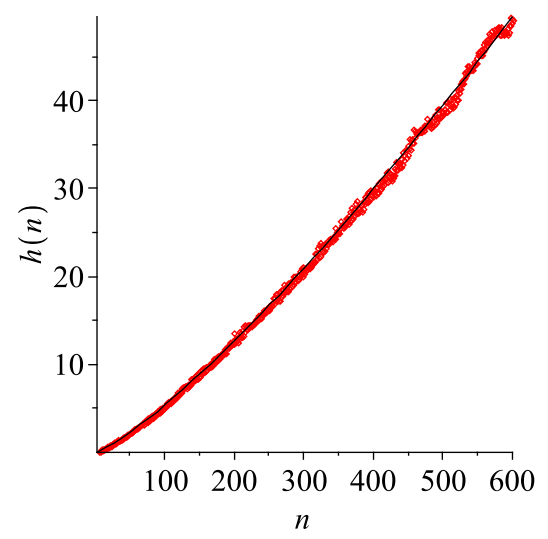

(a)

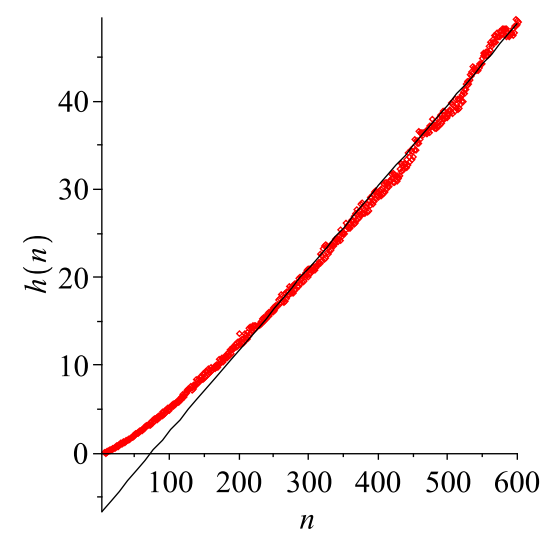

(b)

Figure 17. Least-squares fits of the peak height $h(n)$ of the specific heat $C_{n}\left(\beta_{-}\right)$ of collapsing walks with a negative atmospheric fugacity. The fits are carried out for $n \geq 200$. (a) If one assumes that $h(n)=A n^{2 \phi-1}$ then a regression of the data in Figure 16 gives this left panel with $A=0.0176$ and $\phi=1.12$. (b) If one assumes that $\phi=1$ and $h(n)=A n^{2 \phi-1}+\hat{a}$, then the regression gives the results in this right panel with $A=0.0929$ and $\hat{a}=-6.8425$.

linear regression estimate for $n \geq 200$ of $A \approx 0.0929$ and $\hat{a} \approx-6.8425$. We plot this regression result in Figure 17(b), along with the actual values of the peak heights. The larger value of $\phi$ describes the data better, but we cannot rule out the possibility that $\phi=1$ from our results. The theoretical bound $\phi \leq 1$ implies that our data is consistent with $\phi=1$, and this result suggests a first order transition in this model.

The argument of the scaling function $f$ in equation (12) suggest that the location of the peak in the specific heat curves in Figure 16, denoted by $b(n)$, should move with increasing $n$ at a rate $b(n)=\beta_{-}^{*}+B n^{-\phi}$. Least squares regressions using the data in Figure 16 for $n \geq 200$, assuming that $\phi=1.12$ gives $B=-27.9968$ and $\beta_{-}^{*}=-0.0087$. On the other hand, assuming that $\phi=1$ gives $B=-15.5836$ and $\beta_{-}^{*}=-0.004$. The results of these fits are plotted in Figure 18, along with the actual peak locations.

In the vicinity of the critical point the energy per monomer should scale as

$$
U_{n} \sim n^{\phi-1} f\left(\left(\beta_{-}-\beta_{-}^{*}\right) n^{\phi}\right),
$$

where $f$ is a a scaling function. Thus, plotting $U_{n} n^{1-\phi}$ against $\left(\beta_{-}-\beta_{-}^{*}\right) n^{\phi}$ should collapse data for different values of $n$ to a single curve. In Figure 19 we plot these data for $n \geq 200$. Observe that the curves collapse onto a single curve in Figure 19(b), where we chose the regression estimates $\phi=1$ and $\beta_{-}^{*}=-0.004$.

The specific heat scales with $n$ as in equation (12). Similarly to the energy per monomer, the specific heat data in Figure 16 will collapse to a single curve if one plots $C_{n} n^{1-2 \phi} / A$ as a function of $n^{\phi}\left(\beta_{-}-\beta_{-}^{*}\right)$, where $A$ is a constant. These plots are presented in Figure 20 for $n \geq 200$.

3.3.1. Metric scaling: The scaling of collapsing walks can also be examined by considering the scaling of metric quantities. We focus in particular on the mean 
squared end-to-end distance $\left\langle R_{n}^{2}\left(\beta_{-}\right)\right\rangle$and on the mean squared radius of gyration $\left\langle S_{n}^{2}\left(\beta_{-}\right)\right\rangle$. One expects that the scaling of any quantity with dimensions [length] will be determined by a metric exponent $\nu\left(\beta_{-}\right)$, where $\nu(0)=\nu=3 / 4$ is the exact value of expanded (free) self-avoiding walks in two dimensions [13, 29]. In order to obtain precise estimates of the cross-over exponent and $\beta_{-}^{*}$ we ran two sets of simulations: one that did not measure the radius of gyration and one that did. This is because (as noted earlier) simulations that measure the radius of gyration require $O\left(N^{3}\right)$ time which is considerably slower than the $O\left(N^{2}\right)$ time required by simulations that only measure atmospheric statistics. For the metric data, we also grew walks of lengths up to $n=600$ and collected data in increments of 10 . A total of $2.18 \times 10^{5}$ walks were started, and the pruning and enrichment processes eventually produced a sample of $1.31 \times 10^{10}$ walks for data analysis, of which $1.27 \times 10^{8}$ walks reached the full length.

In Figure 21(a) it appears that the end-to-end distance of walks is close to zero for negative values of $\beta_{-}$. In this compact or globule phase, the endpoints of the walks tend to be close together in space, and this depends only weakly on $n$ (see Figure 7). For positive values of $\beta_{-}$, the end-to-end distances increases proportional to $n^{3 / 4}$; this can be observed in Figure 21(b).

Generally, one may assume that the metric exponent is a function of $\beta_{-}$, in particular, assume that $\left\langle R_{n}^{2}\left(\beta_{-}\right)\right\rangle \sim n^{2 \nu_{R}\left(\beta_{-}\right)}$. One may estimate $\nu_{R}\left(\beta_{-}\right)$by looking at the ratio $\left\langle R_{2 n}^{2}\left(\beta_{-}\right)\right\rangle /\left\langle R_{n}^{2}\left(\beta_{-}\right)\right\rangle$because

$$
\ln \left(\frac{\left\langle R_{2 n}^{2}\left(\beta_{-}\right)\right\rangle}{\left\langle R_{n}^{2}\left(\beta_{-}\right)\right\rangle}\right) / 2 \ln 2=\nu_{R}\left(\beta_{-}\right) .
$$

In Figure 22 we plot these estimates, which show that $\nu_{R}\left(\beta_{-}\right) \approx 3 / 4$ as $\beta_{-}>\beta_{-}^{*}$. The

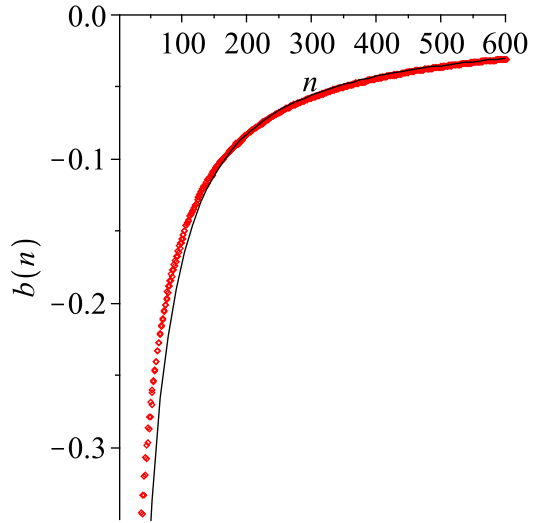

(a)

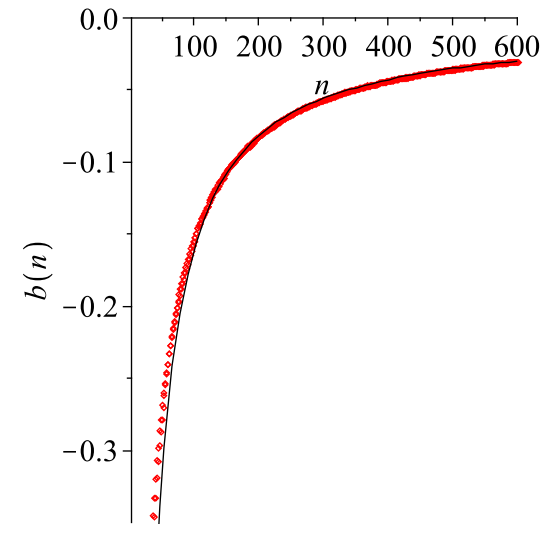

(b)

Figure 18. The locations of the peak in the specific heat data compared with regressions of the location given by $b(n)=\beta_{-}^{*}+B n^{-\phi}$ of the peak locations of the specific heat, based on data from Figure 16. The fits are carried out for $n \geq 200$. On the left panel $(a)$ we assumed that $\phi=1.12$, and obtained $B=-27.9968$ and $\beta_{-}^{*}=-0.0087$. On the right panel $(b)$ we assumed that $\phi=1$, and obtained $B=-15.5836$ and $\beta_{-}^{*}=-0.004$. 


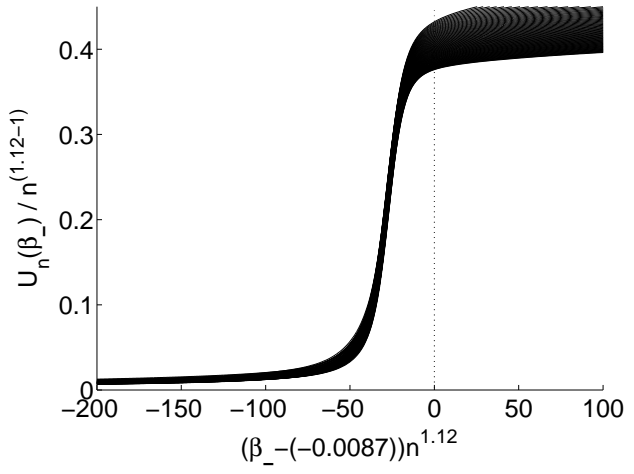

(a)

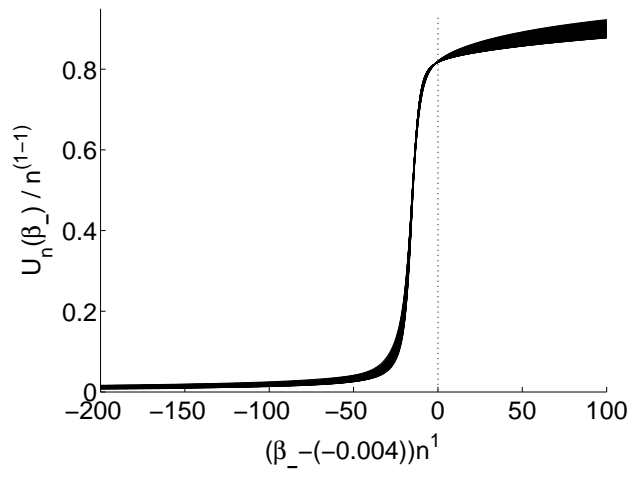

(b)

Figure 19. Scaled internal free energy per monomer $U_{n} n^{1-\phi}$ plotted against $\left(\beta_{-}-\beta_{-}^{*}\right) n^{\phi}$ for $n \geq 200$ with $(a) \phi=1.12$ and $\beta_{-}^{*}=-0.0087$, and $(b) \phi=1$ and $\beta_{-}^{*}=-0.004$.

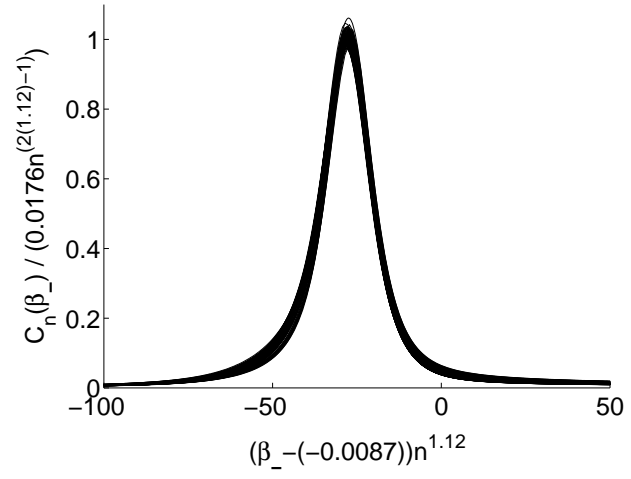

(a)

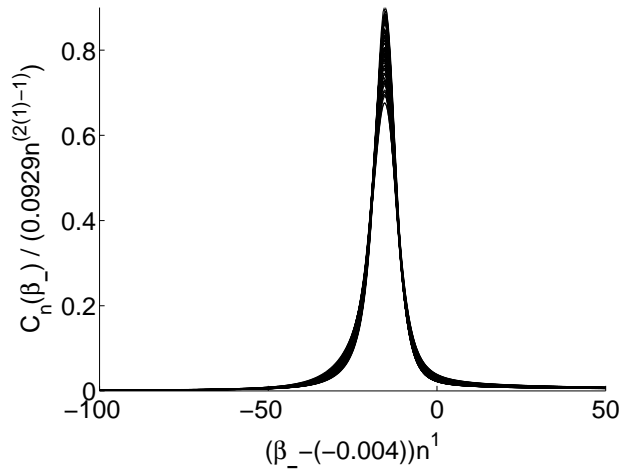

(b)

Figure 20. Scaled specific heat $C_{n} / A n^{2 \phi-1}$ plotted against $\left(\beta_{-}-\beta_{-}^{*}\right) n^{\phi}$ for $n \geq 200$ with (a) $A=0.0176, \phi=1.12$, and $\beta_{-}^{*}=-0.0087$; and $(b) A=0.0929$, $\phi=1$, and $\beta_{-}^{*}=-0.004$.

curves collapse to a single single curve for $\beta_{-}>\beta_{-}^{*}$, but when $\beta_{-}<\beta_{-}^{*}$ these curves accumulate on zero with increasing $n$.

The behaviour of the mean squared radius of gyration is more complex. We examine these statistics in Figures 23( $a$ ) and $(b)$, and estimates for the metric exponent $\nu_{S}\left(\beta_{-}\right)$can be obtained as in Figure 24. The scaling of the radius of gyration is proportional to $n^{3 / 4}$ for values of $\beta_{-}>\beta_{-}^{*}$, as can be seen in Figure $23(b)$.

Estimates of $\nu_{S}\left(\beta_{-}\right)$are plotted against $\beta_{-}$in Figure 24 , and again the data collapse to a single curve for $\beta_{-}>\beta_{-}^{*}$. For smaller values of $\beta_{-}$the behaviour is noisy, but with increasing $n$ settles down near the critical point, while it still retains 
an expanded value for negative values of $\beta_{-}<\beta_{-}^{*}$. The figures of collapsing walks (see Figure 7 ) suggest that the expanded phase is dominated by conformations with $\nu_{S} \approx 3 / 4$, while the compact or globule phase (for negative $\beta_{-}<\beta_{-}^{*}$ ) are instead populated by conformations with endpoints close together. These conformations are polygon-like, and their mean squared radius of gyration scaling may be dominated by conformations with polygon statistics. If this is so, then this phase will have different scaling for end-to-end and mean squared radius of gyration quantities in the compact phase; this is a breakdown of universality in this regime. Comparing Figures 22 and 24 supports this conclusion strongly.

3.3.2. Discussion: Our data suggest the notion that this model has a first order phase transition at the critical point $\beta_{-}=\beta_{-}^{*}$, where the model collapses from a phase of expanded conformations with self-avoiding walk statistics $\left(\beta_{-}>\beta_{-}^{*}\right)$ to a phase of globular or compact conformations dominated by walks with endpoints close together in space. This transition is most clearly seen in the energy per monomer data plotted in Figure 15, which has the appearance of a jump discontinuity at the critical point $\beta_{-}^{*}$. Overall, our data is consistent with a crossover exponent $\phi=1$.

The metric data paints a more complex picture, and in the compact phase we see different scaling behaviour for the mean end-to-end and mean square radius of gyration data.

To gather more evidence for a first order transition in this model, we search our numerical results for evidence of a co-existence of two distinct phases at the critical point $\beta_{-}^{*}$. Our attempts were made difficult with noisy data in the compact phase.

In Figure 25(a) we plot the energy distribution for walks of length $n=600$ and a value of $\beta_{-} \ll \beta_{-}^{*}$. The resulting distribution shows a single dominant peak corresponding to walks with low values of negative atmospheres. This demonstrates a single phase of compact walks.

In Figure 25(b) we plot the energy distribution for walk of length $n=600$ and a value of $\beta_{-} \gg \beta_{-}^{*}$. The resulting distribution shows a single dominant peak

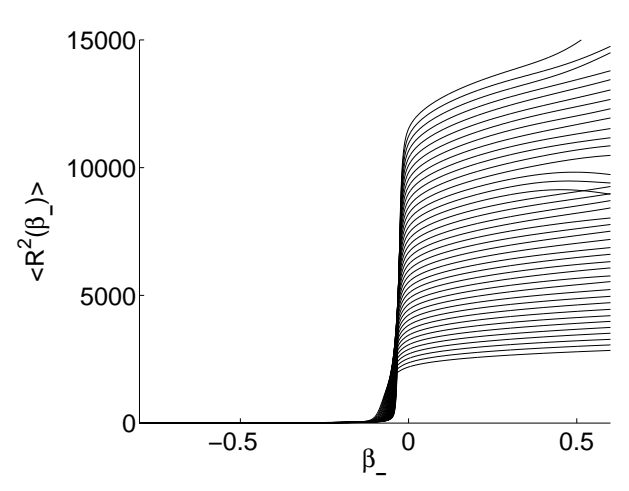

(a)

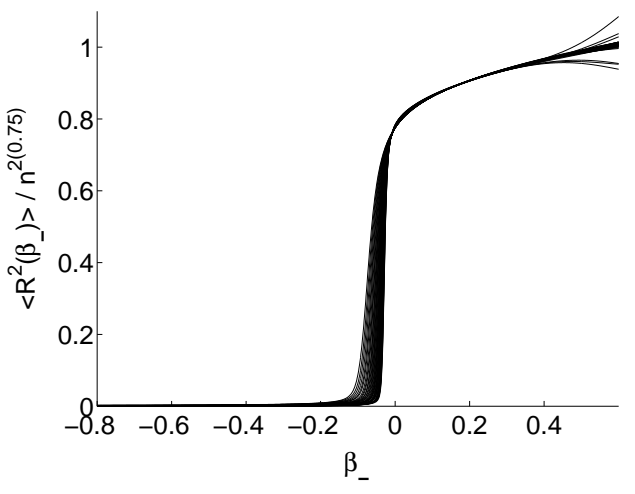

(b)

Figure 21. Mean squared end-to-end distance data. $(a)<R_{n}^{2}\left(\beta_{-}\right)>$. (b) $<R_{n}^{2}\left(\beta_{-}\right)>/ n^{2 \nu_{R}}$ for $\nu_{R}=3 / 4$. 


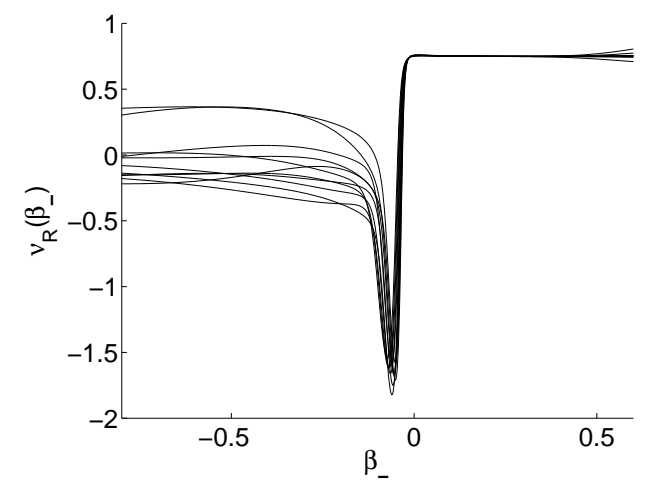

Figure 22. Estimate of $\nu_{R}\left(\beta_{-}\right)$from end-to-end distance, obtained from $\nu_{R}\left(\beta_{-}\right)=\ln \left(\left\langle R_{2 n}^{2}\left(\beta_{-}\right)\right\rangle /\left\langle R_{n}^{2}\left(\beta_{-}\right)\right\rangle\right) / 2 \ln 2$.

corresponding to walks with large values of negative atmospheres. This is the expanded or self-avoiding walk phase in this model, and this shows that a single phase of expanded walks dominate the model in this regime.

The peaks in Figure 25 do not move much with increasing or decreasing values of $\beta_{-}$; instead, they tend to grow or shrink in size. Close to $\beta_{-}^{*}$ more peaks appear in the data between these peaks. This can be seen in Figure 26(a), where the two peaks at 0.05 and 0.90 are still present (and slightly displaced from their extreme positions), but where new peaks at intermediate values of $\beta_{-}$have appeared. These peaks at intermediate values ( 0.3 and 0.45$)$ appear in the data with increasing values of $\beta_{-}$, but vanish once $\beta_{-}>\beta_{-}^{*}$ while only the peak at 0.9 remains. The data plotted in Figure 26(a) show the situation near the critical point (close to the location of the peak in the heat capacity for walks of length $n=600$ ), and the presence of the peaks at the extreme values of the atmospheric statistics can be taken as evidence for the

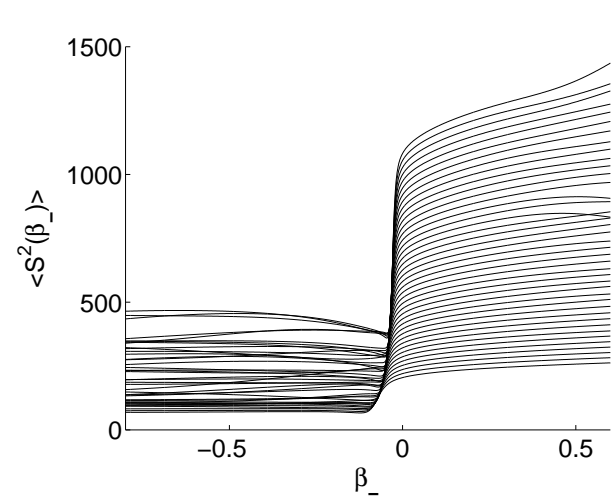

(a)

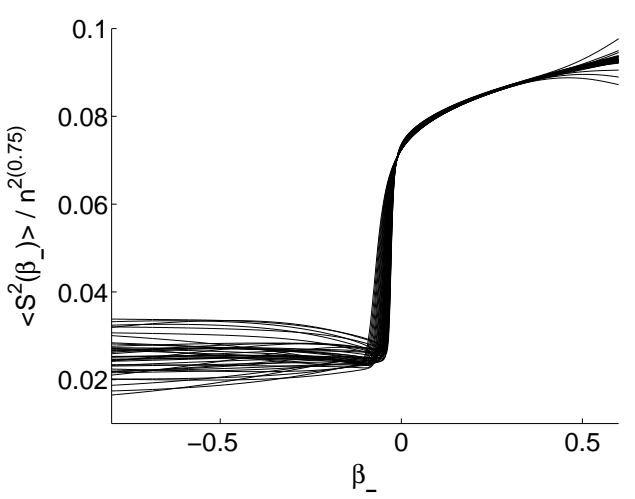

(b)

Figure 23. Mean squared radius of gyration data. $<S_{n}^{2}\left(\beta_{-}\right)>/ n^{2 \nu_{S}}$ for $\nu_{S}=3 / 4$.

$(a)<S_{n}^{2}\left(\beta_{-}\right)>$. (b) 


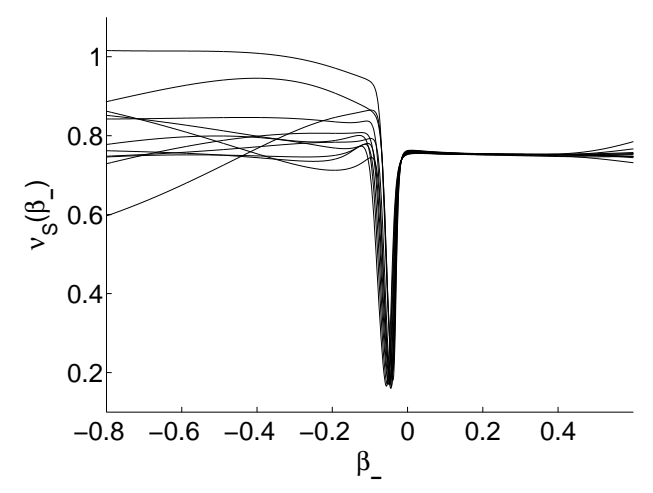

Figure 24. Estimate of $\nu_{S}$ from radius of gyration, obtained from $\nu_{S}\left(\beta_{-}\right)=$ $\ln \left(\left\langle S_{2 n}^{2}\left(\beta_{-}\right)\right\rangle /\left\langle S_{n}^{2}\left(\beta_{-}\right)\right\rangle\right) / 2 \ln 2$.

coexistence of the two phases. The intermediate peaks appear to be noise in the data, however they were quite persistent and repeating our simulations for other values of $n$ or extending the length of the simulations did not substantially change the picture.

In Figures 26(b) and (c) we show plots for the energy distribution for two different values of $\beta_{-}$close to the critical point. In Figure 26(b) we plot the distribution for the value of $\beta_{-}$where the peak from the compact phase and a peak at intermediate energy densities are equal in height. In Figure 26(c) we plot the energy distribution for a value of $\beta_{-}$where the peak at the intermediate energy density dominates both the peaks from both the compact and the extended phase.

The appearance of peaks in the energy distribution between low and high energy phases in Figure 25 together with the jump discontinuity in the energy seen in the rescaled quantities plotted in Figure 19 shows a sharp transition in this model. The scaling of the rescaled energy in Figure 19 and the rescaled specific heat in Figure 20 is

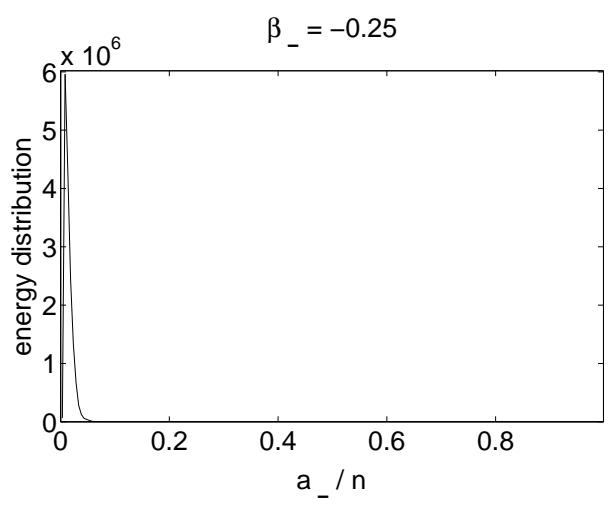

(a)

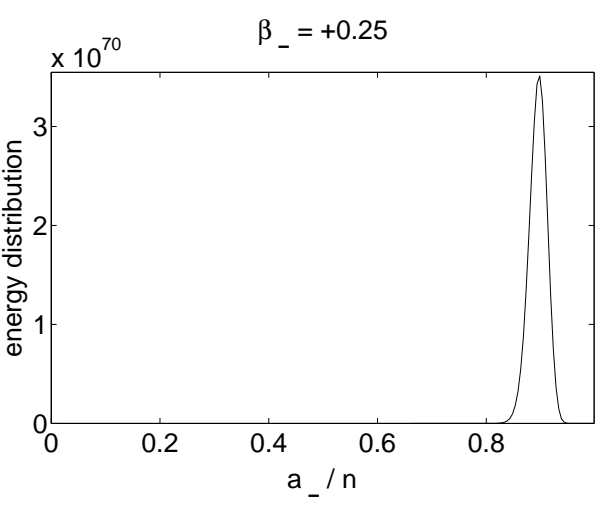

(b)

Figure 25. Energy distribution among states for walks of length $n=600$ and (a) $\beta_{-} \ll \beta_{-}^{*}$, and $(b) \beta_{-} \gg \beta_{-}^{*}$. 


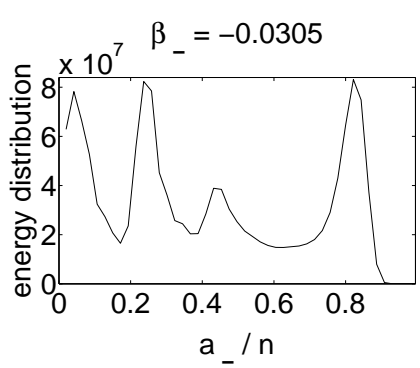

(a)

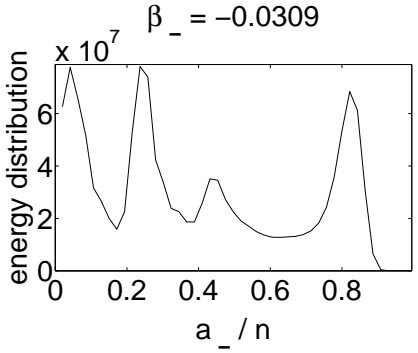

(b)

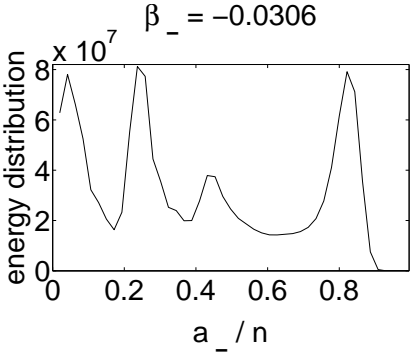

(c)

Figure 26. Energy distribution among states for walks of length $n=600$ and $\beta_{-} \approx \beta_{-}^{*}$.

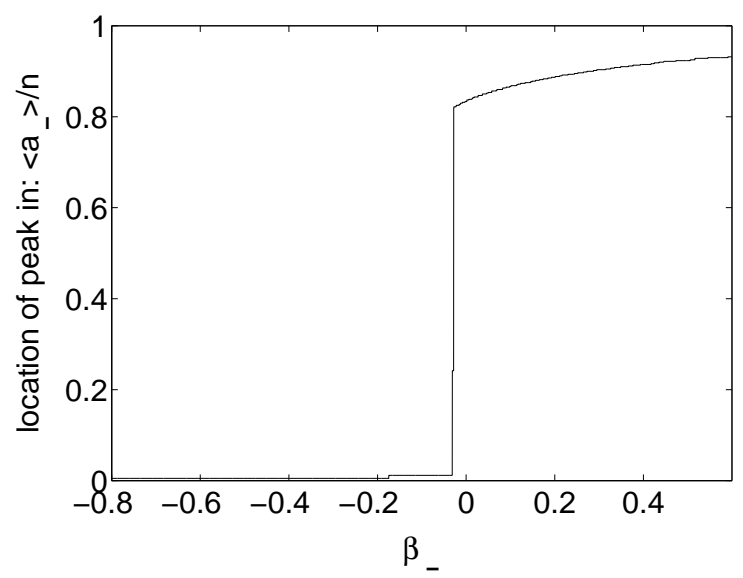

Figure 27. Location of peak in energy distribution per state for negative atmospheric collapse of walks of length $n=600$.

consistent with $\phi=1$, and with a first order transition in this model. This presumption is backed up by the metric data in Figures 22 and 24.

Further evidence can be found by tracking the highest peak in Figure 26 as a function of $\beta_{-}$. For $\beta_{-} \ll \beta_{-}^{*}$ the peak is located at small energy densities, as in Figure 25(a), while for $\beta_{-} \gg \beta_{-}^{*}$ it is located at high energy densities as in Figure 25(b). The switch-over of the dominant peak position at $\beta_{-} \approx \beta_{-}^{*}$ is sudden, and is plotted in Figure 27. This shows a sudden transition from a low energy density state to a high energy density, rather than a continuous transition, which is further evidence suggesting a first order phase behaviour in this model.

\subsection{Positive atmospheric collapse in walks}

Consider a model of positive atmospheric collapse whose partition function is given by $Z_{n}\left(\beta_{+}\right) \equiv Z_{n}\left(\beta_{+}, 0,0\right)$ (see equation (7)). The behaviour of this model is qualitatively similar to that of the negative generalised atmospheres (Section 3.3). Large negative 
values of $\beta_{+}$are characterised by conformations which appear compact, while positive values of $\beta_{+}$are characterised by conformations which appear to be expanded. Typical conformations of two-dimensional walks with small and large positive atmospheres are depicted in Figures 4 and 5.

In Theorem 3.2 the limiting intensive free energy of this model is given by $\mathcal{F}\left(\beta_{+}\right) \equiv \mathcal{F}\left(\beta_{+}, 0,0\right)=\lim _{n \rightarrow \infty}=n^{-1} \log Z_{n}\left(\beta_{+}\right)$and this exists for all values of $\beta_{+} \geq 0$. The existence of a limiting free energy in this model for $\beta_{+}<0$ remains an open question.

Numerical data, analogous to those for presented for negative atmospheric collapse (Section 3.3), were collected for two-dimensional walks of length $n$ from $n=100$ to $n=600$. These data were obtained using flatGARM with $1.12 \times 10^{5}$ started walks, and the pruning and enrichment processes eventually produced a sample of $2.49 \times 10^{10}$ walks for data analysis, of which $2.18 \times 10^{8}$ walks reached the full length.

In Figure 28(a) we plot the intensive free energy $\mathcal{F}_{n}\left(\beta_{+}\right)$. There appears to be a sharp transition around $\beta_{+}^{*} \approx 0$, below which the curves increase with $n$ to an apparently horizontal limiting curve, and above which the free energy curves collapse onto a single curve that appears to increase linearly with $\beta_{+}$.

This suggests a first order phase transition similar to the transition in negative atmospheric collapsing walks. The internal energy per monomer, $U_{n}\left(\beta_{+}\right)$, accumulates with increasing $n$ on a limiting curve with a jump discontinuity consistent with a first order phase transition, as can be observed in Figure 28(b). The location of the transition between the compact and the extended phases is approximated by the vertical part of the curve.

The specific heat for finite length $n, C_{n}\left(\beta_{+}\right)$, diverges near the transition point $\beta_{+}^{*}$ as $n$ increases, as can be observed in Figure 28(c).

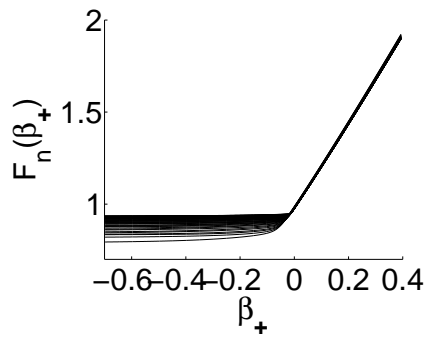

(a)

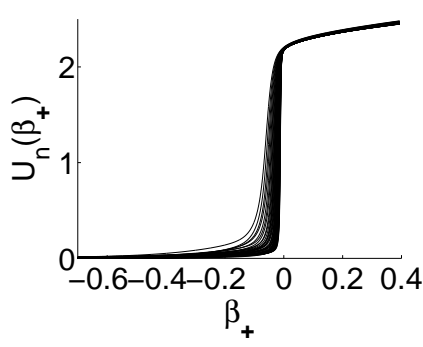

(b)

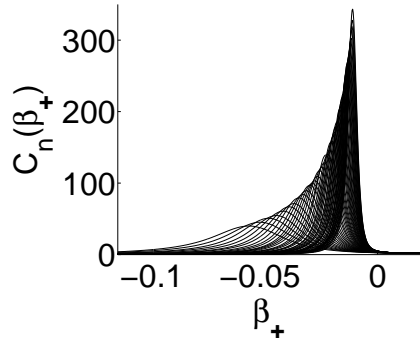

(c)

Figure 28. Two-dimensional positive atmospheric collapse. (a) Intensive free energy, $\mathcal{F}_{n}\left(\beta_{+}\right)$. (b) Internal energy per monomer, $U_{n}\left(\beta_{+}\right)$. (c) Specific heat, $C_{n}\left(\beta_{+}\right)$.

We investigated the critical scaling of the specific heat (see the discussion around Equation (12)) for this positive atmospheric collapse model. The height of the peak in $C_{n}\left(\beta_{+}\right)$was used to determine the crossover exponent $\phi$ from the data in Figure 28(c). The resulting least squares fit of the form $A n^{2 \phi-1}$ suggests that $A \approx 0.2344$ and $\phi \approx 1.07$. Values of $\phi$ larger than 1 and strong correction to scaling in our data indicated that we cannot rule out that $\phi=1$. Assuming that $\phi=1$ and then fitting our data to $A n^{2 \phi-1}+\hat{a}$ produced $A \approx 0.599$ and $\hat{a} \approx-24.8169$. 
We also analysed the location of the peak in $C_{n}\left(\beta_{+}\right)$by regressions against $\beta_{+}^{*}+B n^{-\phi}$. Assuming that $\phi=1.07$ gives $B=-8.3324$ and $\beta_{+}^{*}=-0.0027$. On the other hand, assuming that $\phi=1$ gives $B=-5.9799$ and $\beta_{+}^{*}=-0.0016$. These results are consistent with $\phi=1$.

In the vicinity of the critical point, the internal energy per monomer should scale as Equation (13). Thus, plotting $U_{n} n^{1-\phi}$ against $\left(\beta_{+}-\beta_{+}^{*}\right) n^{\phi}$ should collapse data for different values of $n$ to a single curve. Figure 29 plots the scaled internal energy per monomer. Observe that when $\left(\beta_{+}-\beta_{+}^{*}\right) n^{\phi}=0$ the curves collapse onto a single curve in Figure 29(b), where we chose the regression estimates $\phi=1$ and $\beta_{-}^{*}=-0.0016$.

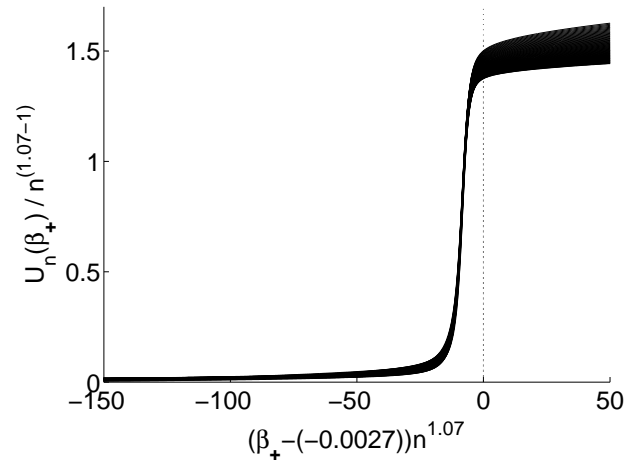

(a)

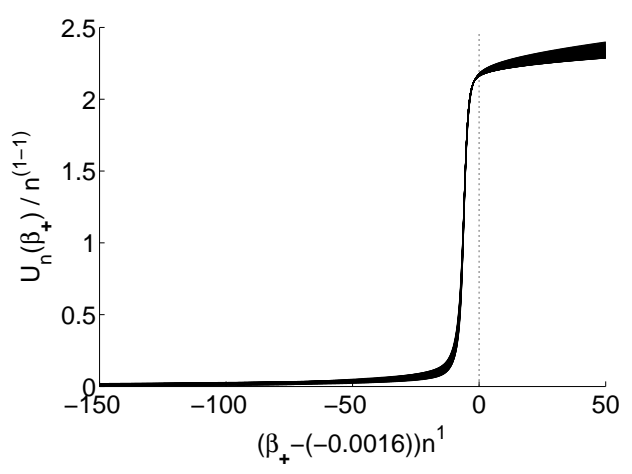

(b)

Figure 29. Scaled internal free energy per monomer $U_{n} n^{1-\phi}\left(\beta_{+}\right)$plotted against $\left(\beta_{+}-\beta_{+}^{*}\right) n^{\phi}$ for $n \geq 200$ with $(a) \phi=1.07$ and $\beta_{+}^{*}=-0.0027$, and $(b) \phi=1$ and $\beta_{+}^{*}=-0.0016$.

One can again analyse the scaling by considering metric quantities like the mean squared end-to-end distance $\left\langle R_{n}^{2}\left(\beta_{+}\right)>\right.$, whose scaling is expected to be determined by a metric exponent $\nu_{R}\left(\beta_{+}\right)$. One may estimate $\nu_{R}\left(\beta_{+}\right)$by looking at the ratio $\left\langle R_{2 n}^{2}\left(\beta_{+}\right)\right\rangle /\left\langle R_{n}^{2}\left(\beta_{+}\right)\right\rangle$, as indicated by Equation (14). The metric data were obtained using flatGARM with $6.8 \times 10^{4}$ started walks, and the pruning and enrichment processes eventually produced a sample of $1.54 \times 10^{10}$ walks for data analysis, of which $1.37 \times 10^{8}$ walks reached the full length.

It appears that the mean squared end-to-end distance $\left\langle R_{n}^{2}\left(\beta_{+}\right)\right\rangle$of the twodimensional positive atmospheric collapse is close to zero for large negative values of $\beta_{+}$(see Figure 30(a)). In this compact or globule phase, the endpoints of the walks tend to be close together in space, and this depends only weakly on $n$ (see Figure 4). For positive values of $\beta_{+}$, the mean squared end-to-end distance increases proportional to $n^{3 / 4}$, as can be seen in Figures $30(b)$ and $(c)$.

The behaviour of the mean squared radius of gyration is again slightly more complex, as observed in Figure 31. For values of $\beta_{+}>\beta_{+}^{*}$ the scaling of the radius of gyration is proportional to $n^{3 / 4}$, as can be seen in Figures $31(b)$ and $(c)$. For smaller values of $\beta_{+}$the behaviour is noisy, but as $n$ increases it settles down near the critical point, while it still retains an expanded value for negative values of $\beta_{+}<\beta_{+}^{*}$. The figures of collapsing walks (see Figure 4) suggest that the expanded phase is dominated by conformations with $\nu_{S} \approx 3 / 4$, while the compact or globule phase 


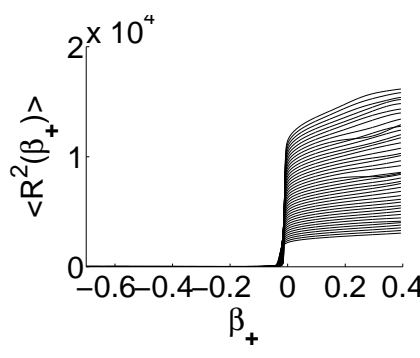

(a)

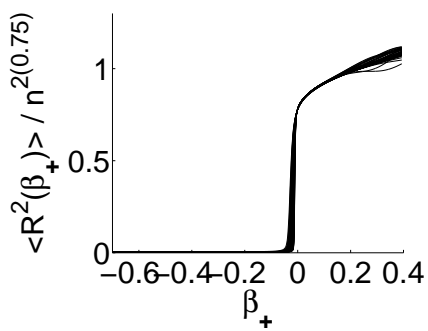

(b)

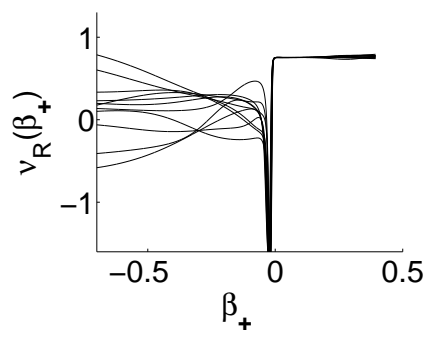

(c)

Figure 30. Mean squared end-to-end distance data. $(a)<R_{n}^{2}\left(\beta_{+}\right)>$. (b) $<R_{n}^{2}\left(\beta_{+}\right)>/ n^{2 \nu_{R}}$ for $\nu_{R}=3 / 4$. (c) Estimate of $\nu_{R}\left(\beta_{+}\right)$from end-to-end distance, obtained from $\nu_{R}\left(\beta_{+}\right)=\ln \left(\left\langle R_{2 n}^{2}\left(\beta_{+}\right)\right\rangle /\left\langle R_{n}^{2}\left(\beta_{+}\right)\right\rangle\right) / 2 \ln 2$.

(for negative $\beta_{+}<\beta_{+}^{*}$ ) are instead populated by conformations with endpoints close together. These conformations are polygon-like, and their mean squared radius of gyration scaling may be dominated by conformations with polygon statistics. If this is so, then this phase will have different scaling for end-to-end and mean squared radius of gyration quantities in the compact phase; this is a breakdown of universality in this regime. Comparing Figures 30 and 31 supports this conclusion strongly.

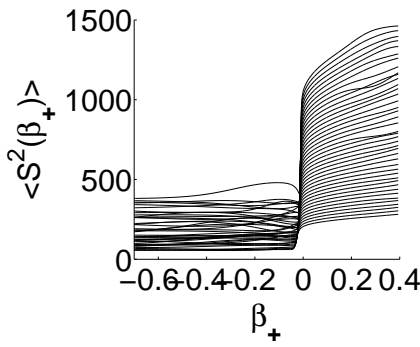

(a)

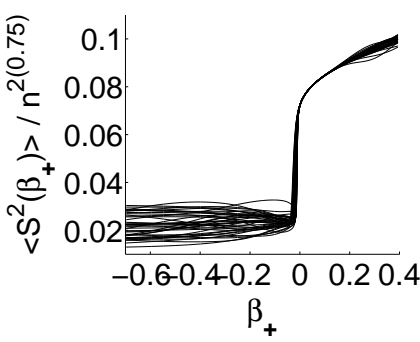

(b)

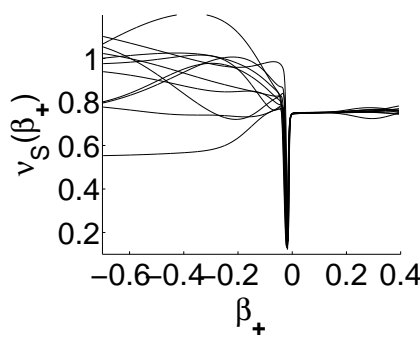

(c)

Figure 31. Mean squared radius of gyration data. $(a)<S_{n}^{2}\left(\beta_{+}\right)>$. (b) $<S_{n}^{2}\left(\beta_{+}\right)>/ n^{2 \nu_{S}}$ for $\nu_{S}=3 / 4$. (c) Estimate of $\nu_{S}$ from radius of gyration, obtained from $\nu_{S}\left(\beta_{+}\right)=\ln \left(\left\langle S_{2 n}^{2}\left(\beta_{+}\right)\right\rangle /\left\langle S_{n}^{2}\left(\beta_{+}\right)\right\rangle\right) / 2 \ln 2$.

As in the case of negative atmospheric collapse (Section 3.3), our data suggest that the model has a first order transition at the critical point $\beta_{+}=\beta_{+}^{*}$, where the model collapses from a phase of expanded conformations with self-avoiding walk statistics $\left(\beta_{+}>\beta_{+}^{*}\right)$ to a phase of globular or compact conformations dominated by walks with endpoints close together in space. Overall, our data is consistent with a crossover exponent $\phi=1$.

We searched our numerical results for evidence of a co-existence of two distinct phases at the critical point $\beta_{+}^{*}$, and again, our attempts were made difficult by noisy data in the compact phase. In particular, we analysed the energy distribution for 
walks as a function of $\beta_{+}$. A single dominant peak in the energy distribution indicates the dominance of one phase over the other, while the co-existence of the two phases would be characterised by a pair of co-dominant peaks.

Figure 32 plots the location of the dominant peak(s) of the energy distribution. For $\beta_{+} \ll \beta_{+}^{*}$ the peak is located at small energy densities, while for $\beta_{+} \gg \beta_{+}^{*}$ it is located at high energy densities. The switch-over of the dominant peak position at $\beta_{+} \approx \beta_{+}^{*}$ is sudden. This shows a sudden transition from a low energy density state to a high energy density, rather than a continuous transition. This is further evidence suggesting a first order phase behaviour in this model.

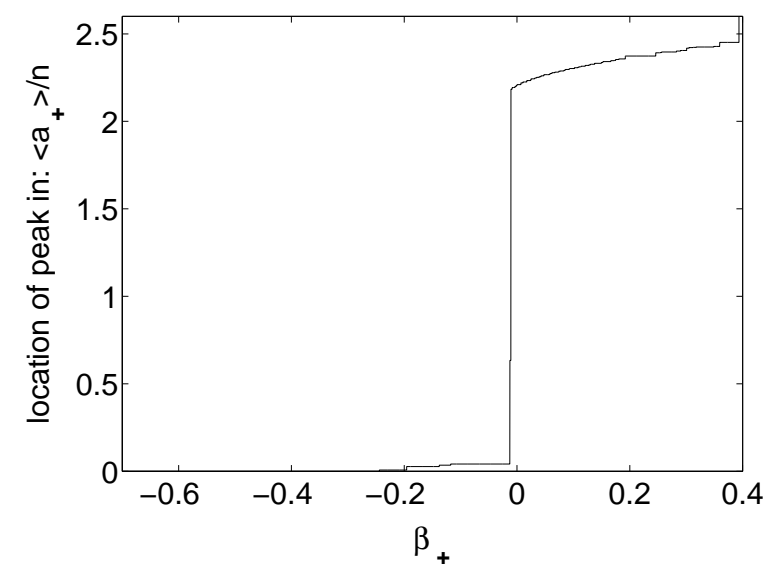

Figure 32. Location of peak in energy distribution per state for positive atmospheric collapse walks of length $n=600$.

\subsection{Neutral atmospheric collapse}

In this section we examine neutral atmospheric collapse in self-avoiding walks. The partition partition function of this model is given by $Z_{n}\left(\beta_{0}\right) \equiv Z_{n}\left(0, \beta_{0}, 0\right)$ (see Equation (7)). We collected numerical results for the neutral atmospheric collapse of two-dimensional walks of lengths $n=100$ to $n=600$ using flatGARM with a total of $2.32 \times 10^{5}$ started walks. The pruning and enrichment processes eventually produced a sample of $2.82 \times 10^{10}$ walks for data analysis, of which $1.92 \times 10^{8}$ walks reached the full length.

For large positive values of $\beta_{0}$ the model is dominated by walks with many $90^{\circ}$ turns and staircase-like conformations, as illustrated in Figure 10. This is an expanded regime of walks. For large negative values of $\beta_{0}$, the model is dominated by walks with few $90^{\circ}$ turns and a layered appearance, as illustrated in Figure 11. We refer to this as a layered regime.

As indicated by Theorem 3.2, the limiting free energy of this model $\mathcal{F}_{n}\left(\beta_{0}\right) \equiv$ $\mathcal{F}_{n}\left(0, \beta_{0}, 0\right)=\lim _{n \rightarrow \infty} n^{-1} \log Z_{n}\left(\beta_{0}\right)$ exists for all values of $\beta_{0} \geq 0$.

In Figure 33(a) we plot the intensive free energy $\mathcal{F}_{n}\left(\beta_{0}\right)$ as a function of $\beta_{0}$. The curves are smooth functions of $\beta_{0}$, with no evident singularities suggesting a phase transition in this model.

The internal energy per monomer, defined by $U_{n}\left(\beta_{0}\right)=\left\langle a_{0}^{g}\right\rangle / n$ is plotted in Figure $33(b)$ and the specific heat $C_{n}\left(\beta_{0}\right)=\left[\left\langle\left(a_{0}^{g}\right)^{2}\right\rangle-\left\langle a_{0}^{g}\right\rangle^{2}\right] / n$ is plotted in 


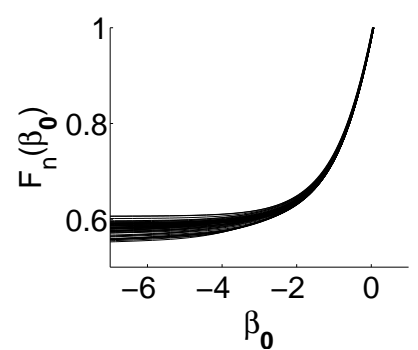

(a)

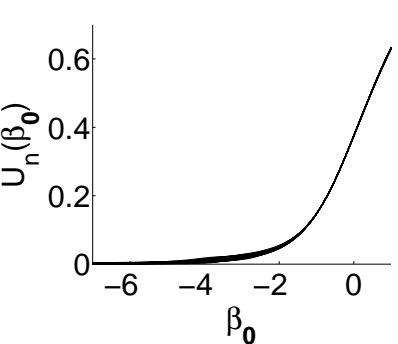

(b)

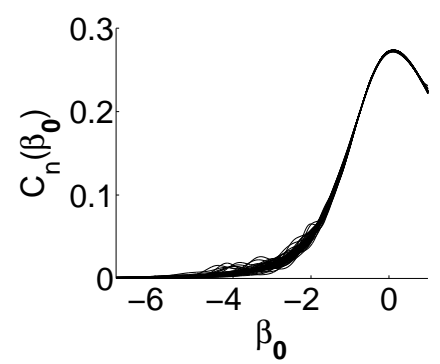

(c)

Figure 33. Two-dimensional neutral atmospheric collapse. (a) Intensive free energy, $\mathcal{F}_{n}\left(\beta_{0}\right)$. (b) Internal energy per monomer, $U_{n}\left(\beta_{0}\right)$. (c) Specific heat, $C_{n}\left(\beta_{0}\right)$.

Figure 33(c). It appears that there is no increase in height or width of the peak in $C_{n}\left(\beta_{0}\right)$ with $n$. There is no evidence of critical scaling in the specific heat: Both the location of the peak, and the width of the peak, are insensitive to $n$. We also carried out a least-squares fit to the peaks of the specific heat and obtained a value of crossover exponent $\phi \approx 0.5$, which is consistent with no transition. Hence, we conclude that there is no critical behaviour in this model, and the crossover from the layered to an expanded regime is not a critical phenomenon. This is similar to observations made in other models of self-avoiding walks, for example, in models of walks and lattice ribbons examined for critical behaviour in torsion and twist statistics; see references $[23,30,31]$.

\section{Conclusions}

We analysed several models of self-avoiding walk collapse based on endpoint and generalised atmospheric statistics. The collapse is essentially driven by confining or limiting the walk to states in conformational space with low degrees of freedom, as it occurs in the case of the $\theta$ - and $\theta^{\prime}$ - models.

We proved that the limiting free energy of collapse driven by endpoint atmospheres exists over the entire parameter space and that it is equal to the logarithm of the connective constant for self-avoiding walks.

We also proved that the limiting free energy of collapse driven by generalised atmospheres exists for non-negative values of the parameters. The existence of the limiting free energy remains unknown if at least one of the parameters is negative. This situation is analogous to other collapsing walk models (like nearest neighbour contacts).

We carried out a numerical analysis of the phase transition in these models of generalised atmospheres using the flatGARM algorithm [17]. Our analysis indicates that the models of collapse driven by negative generalised atmospheres and by positive generalised atmospheres exhibit a strong transition separating an expanded phase from a compact phase, which is characterised by walks with endpoints close together in space, illustrated in Figures 4 and 7 . Our calculations for those two models yielded thermodynamic behaviour and crossover exponents consistent with a first order 
transition.

The internal energy per monomer approaches a discontinuous function as the length of the walks increases, the peak heights of the specific heat diverge near the location of the discontinuity, and it appears that the energy distribution consists of a pair of co-existent peaks near that point as well (although our data remains very noisy in the compact phase). We also considered the metric scaling of walks in these models. The expanded phase is dominated by conformations with their endpoints far apart, while the compact phase is populated by conformations with their endpoints close together. These compact conformations are polygon-like, and they have different scaling for their end-to-end and their mean squared radius of gyration.

We also analysed a model of collapse driven by neutral generalised atmospheres. This model differs significantly from the the other two and we find no evidence for a first order transition. Indeed we find little evidence for any sort of critical behaviour in this model.

A comparison of our results to the results in the literature for the $\theta$ - and $\theta^{\prime}$ transitions in models of collapsing walks shows that the atmospheric collapsing walks in this paper do not belong to the $\theta$-universality class. In particular, atmospheric collapse driven by positive or negative generalised atmospheres appears to be a first order phase transition, while the $\theta$ - and $\theta^{\prime}$-transitions are continuous phase transitions. Overall the numerical simulations in our atmospheric collapse models proved difficult, and required tremendous numerical resources. Further examination of these models will improve our data, and should provide more evidence of a first order phase change in these models.

\section{Acknowledgments}

EJJvR and AR acknowledge financial support from NSERC (Canada). We are thankful to the anonymous referee for the helpful comments.

\section{References}

[1] Aragão de Carvalho C, Caracciolo S and Fröhlich J 1983 Polymers and $g|\phi|^{4}$-theory in Four Dimensions, Nucl. Phys. B215 [FS7] 209-248

[2] Baiesi M, Orlandini E and Stella A L 2006 Scaling of a Collapsed Polymer Globule in Two Dimensions. Phys. Rev. Let. 96 040602-1-4

[3] Barkema G T, Bastolla U, and Grassberger P 1998 Two-Dimensional Oriented Self-Avoiding Walks with Parallel Contacts, J. Stat. Phys. 90, 1311-1324

[4] Berg B and Foester D 1981 Random Paths and Random Surfaces on a Digital Computer, Phys. Lett. 106B 323-326

[5] Berretti A and Sokal A D 1985 New Monte Carlo Method for the Self-Avoiding Walk. J. Stat. Phys. 40 483-531

[6] Brak R, Guttmann A J and Whittington S G 1991 On the Behaviour of Collapsing Linear and Branched Polymers. J. Math. Chem. 8 255-267

[7] Challa M S S, Landau D P, and Binder K 1986 Finite-size effects at temperature-driven firstorder transitions. Phys. Rev. B 34 1841-1852

[8] Chang I S and Meirovitch H 1993 Collapse Transition of Self-avoiding Walks on a Square Lattice in Bulk and near a Linear Wall: The Universality Classes of the $\Theta$ and $\Theta^{\prime}$ Points. Phys. Rev. E 48 3656-3660

[9] Coniglio N, Jan N, Majid I and Stanley H E 1988 Conformation of a Polymer at the $\theta^{\prime}$ Point: Connection to the External Perimeter of a Percolation Cluster. Phys. Rev. B 35 3617-3620

[10] de Gennes P-G 1975 Collapse of a Polymer Chain in Poor Solvents. J. de Physique 36 L55-L57

[11] de Gennes P-G 1979 Scaling Concepts in Polymer Physics. Cornell University Press (Ithaca). 
[12] Duplantier B 1987 Geometry of Polymer Chains near the $\theta$-Point and Dimensional Regularisation. J. Chem. Phys. 86 4233-4244

[13] Duplantier B 1990 Renormalisation and Conformal Invariance for Polymers. In Fundamental Problems in Statistical Mechanics VII, 171-223. Ed. H. van Beijeren (Elsevier: Amsterdam)

[14] Duplantier B and Saleur H 1987 Exact Tricritical Exponents for Polymers at the $\Theta$-Point in Two Dimensions. Phys. Rev. Lett. 59 539-542

[15] Duplantier B and Saleur H 1988 Reply to: Universality Classes for the $\theta$ and $\theta^{\prime}$ Points. Phys. Rev. Lett. 60 1204-1204

[16] Foster D, Orlandini E and Tesi M C 1992 Surface Critical Exponents for Models of Polymer Collapse and Adsorption: The Universality of the $\theta$ - and $\theta^{\prime}$-Points. J. Phys. A: Math. Gen. 25 1211-1217

[17] Grassberger P 1997 Pruned-Enriched Rosenbluth method: Simulations of $\theta$ polymers of chain length up to 1000000 Phys Rev E 56 3682-3693

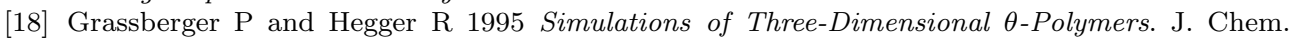
Phys. 102 6881-6899

[19] Grassberger P and Hegger R 1995 Simulations of $\theta$-Polymers in 2 Dimensions. J. de Physique 5, 597-606

[20] Hammersley S J and Welsh D J A 1962 Further Results on the Rate of Convergence to the Connective Constant of the Hypercubical Lattice. Quart. J. Math. (Oxford) 13 108-110

[21] Hille E 1948 Functional Analysis and Semi-Groups. AMS Colloq. Publ. 31 (AMS: Providence, $\mathrm{RI})$

[22] Hsu H-P and Grassberger P 2005 The Coil-Globule Transition of Confined Polymers. J. Stat. Mech.: Theo. Expr. 1 P01007-1-9

[23] Janse van Rensburg E J, Orlandini E, Sumners D W, Tesi M C and Whittington S G 1994 Lattice Ribbons: A Model of Double-Stranded Polymers. Phys. Rev. E50 4279-4283

[24] Janse van Rensburg E J and Rechnitzer A 2008 Atmospheres of Polygons and Knotted Polygons. J. Phys. A: Math. Theo. 41 105002-105025

[25] Janse van Rensburg E J and Rechnitzer R 2008 Generalised Atmospheric Sampling of SelfAvoiding Walks. Preprint.

[26] Jensen I 2003 A Parallel Algorithm for the Enumeration of Self-Avoiding Polygons on the Square Lattice. J. Phys. A: Math. Gen. 36 5731-5745

[27] Maes D and Vanderzande C 1990 Self-Avoiding Rings at the $\Theta$ Point. Phys. Rev. A41 3074-3080

[28] Meirovitch H and Lim H A 1989 The Collapse Transition of Self-Avoiding Walks on a Square Lattice: A Computer Simulation Study. J. Chem. Phys. 91 2544-2554

[29] Nienhuis B 1984 Coulomb Gas Formulation of the Two-Dimensional Phase Transitions. In Phase Transitions and Critical Phenomena 11 1-53. Eds. C. Domb and J.L. Lebowitz (Academic Press)

[30] Orlandini E, Tesi M C, Janse van Rensburg E J and Whittington S G 1997 The Shapes of Self-avoiding Polygons with Torsion, J. Phys. A: Math. Gen. 30 L693-698

[31] Orlandini E, Tesi M C, Whittington S G, Sumners D W and Janse van Rensburg E J 1994 The writhe of a Self-avoiding Walk, J. Phys. A: Math. Gen. 27 L333-L338

[32] Owczarek A L, Prellberg T and Brak R 1993 New Scaling Form for the Collapsed Polymer Phase. Phys. Rev. Lett. 70 951-953

[33] Owczarek A L, Prellberg T and Brak R 1993 Reply: New Scaling Form for the Collapsed Polymer Phase. Phys. Rev. Lett. 71 4275-4275

[34] Poole P H, Coniglio N, Jan N and Stanley H E 1988 Universality Classes for the $\theta$ and $\theta^{\prime}$ Points. Phys. Rev. Lett. 60 1203-1203

[35] Poole P H, Coniglio A, Jan N, and Stanley H E 1989 Universality classes of the $\theta$ and $\theta^{\prime}$ points Phys. Rev. B 39 495-504.

[36] Prellberg T and Krawczyk J 2004 Flat Histogram Version of the Pruned and Enriched Rosenbluth Method. Phys. Rev. Lett. 92 120602-120605

[37] Prellberg T and Owczarek A L 2000 Four-dimensional Polymer Collapse: Pseudo-first-order Transition in Interacting Self-Avoiding Walks. Phys. Rev. E 62 3780-3789

[38] Privman V 1986 Study of the $\theta$-Point by Enumeration of Self-Avoiding Walks on the Triangular Lattice. J. Phys. A: Math. Gen. $193287-3297$

[39] Rechnitzer A and Janse van Rensburg E J 2002 Canonical Monte Carlo Determination of the Connective Constant of Self-Avoiding Walks. J. Phys. A: Math. Gen. 35 L605-L612

[40] Rechnitzer A and Janse van Rensburg E J 2008 Generalised Atmospheric Rosenbluth Methods (GARM). J. Phys. A: Math. Theo. 41 442002-442010

[41] Saleur H 1986 Collapse of Two-Dimensional Linear Polymers. J. Stat. Phys. 45 419-438

[42] Seno F and Stella A L 1988 -Point of a Linear Polymer in 2 Dimensions: A Renormalization 
Group Analysis of Monte Carlo Enumerations. J. de Physique 49 739-748

[43] Seno F and Stella A L 1988 Surface Exponents for a Linear Polymer at the $d=2$ Theta Point. Europhys. Lett. 7 605-610

[44] Seno F, Stella A L and Vanderzande C 1988 Universality Class of the $d=2$-Point of Linear Polymers. Phys. Rev. Lett. 61 1520-1520

[45] Sun S-T, Nishio I, Swislow G and Tanaka T 1980 The CoilGlobule Transition: Radius of Gyration of Polystyrene in Cyclohexane. J. Chem. Phys. 73, 5971-5975

[46] Tesi M C, Janse van Rensburg E J, Orlandini E and Whittington S G 1995 Interacting SelfAvoiding Walks and Polygons in Three Dimensions. J. Phys. A: Math. Gen. 29 2451-2463

[47] Tesi M C, Janse van Rensburg E J, Orlandini E and Whittington S G 1996 Monte Carlo study of the Interacting Self-avoiding Walk Model in Three Dimensions. J. Stat. Phys. 82 155-181

[48] Vanderzande C, Stella A L and Seno F 1991 Percolation, The Special $\theta^{\prime}$ Point, and the $\theta-\theta^{\prime}$ Universality Puzzle. Phys. Rev. Lett. 67 2757-2760 